\title{
Nonstationary Spectral Analysis Based on Time-Frequency Operator Symbols and Underspread Approximations
}

\author{
Gerald Matz, Member, IEEE, and Franz Hlawatsch, Senior Member, IEEE
}

\begin{abstract}
We present a unified framework for time-varying or time-frequency (TF) spectra of nonstationary random processes in terms of TF operator symbols. We provide axiomatic definitions and TF operator symbol formulations for two broad classes of TF spectra, one of which is new. These classes contain all major existing TF spectra such as the Wigner-Ville, evolutionary, instantaneous power, and physical spectrum. Our subsequent analysis focuses on the practically important case of nonstationary processes with negligible high-lag TF correlations (so-called underspread processes). We demonstrate that for underspread processes all TF spectra yield effectively identical results and satisfy several desirable properties at least approximately. We also show that Gabor frames provide approximate Karhunen-Loève (KL) functions of underspread processes and TF spectra provide a corresponding approximate KL spectrum. Finally, we formulate simple approximate input-output relations for the TF spectra of underspread processes that are passed through underspread linear timevarying systems. All approximations are substantiated mathematically by upper bounds on the associated approximation errors. Our results establish a TF calculus for the second-order analysis and time-varying filtering of underspread processes that is as simple as the conventional spectral calculus for stationary processes.
\end{abstract}

Index Terms-Evolutionary spectrum, Gabor expansion, instantaneous power spectrum, Karhunen-Loève (KL) expansion, nonstationary random processes, nonstationary spectral analysis, time-frequency (TF) analysis, time-varying systems, Wigner-Ville spectrum.

\section{INTRODUCTION}

$\mathbf{N}$ ONSTATIONARY random processes are useful models for signals and interference arising in speech and audio, communications, image processing, computer vision, biomedical engineering, machine monitoring, and many other applications. They constitute a much more general theoretical framework than do stationary processes, but they are also much more difficult to describe, analyze, and process. For stationary processes, the power spectral density (PSD) provides a simple, intuitively appealing, and powerful tool for the purposes of description, analysis, and processing. For nonstationary processes, a fully equivalent concept is not available.

Manuscript received May 9, 2003; revised September 23, 2005. This work was supported by Fonds zur Förderung der wissenschaftlichen Forschung (FWF) under Grants P15156 and J2302. The material in this paper was presented in part at the 32nd Asilomar Conference on Signals, Systems, and Computers, Pacific Grove, CA, October 1998.

The authors are with the Institute of Communications and Radio-Frequency Engineering, Vienna University of Technology, A-1040 Vienna, Austria (e-mail: gmatz@nt.tuwien.ac.at; fhlawats@pop.tuwien.ac.at).

Communicated by G. Battail, Associate Editor At Large.

Digital Object Identifier 10.1109/TIT.2005.864419
In an attempt to extend the PSD to nonstationary processes, different definitions of "time-dependent (or time-varying) power spectra" have been proposed over the years, which resulted in a wealth of literature on the topic (e.g., [1]-[24]). Since these spectra describe the process' second-order statistics as a function of time and frequency or, equivalently, over the time-frequency (TF) plane, we here prefer the terminology $T F$ spectra. The major definitions of TF spectra extend specific properties of the PSD to the nonstationary case. However, no single TF spectrum satisfies all desirable properties and thus is satisfactory in all respects.

In this paper, we take a new look at nonstationary spectral analysis and TF spectra. We consider the practically important class of underspread processes, which are nonstationary processes with negligible high-lag TF correlations [25]-[32]. Our principal goal is to develop a simple and powerful TF calculus for the analysis and filtering of underspread processes. This calculus is based on unified formulations of second-order TF spectra in terms of linear TF operator symbols. Specifically, we will show the following.

- TF spectral analysis furnishes satisfactory results only for underspread processes. In contrast, the TF spectra of processes that are overspread (i.e., not underspread) either contain "statistical cross terms" that drastically limit their readability and usefulness [27], [29]-[33], or do not convey the complete information about the process' second-order statistics.

- For underspread processes, all TF spectra satisfy various desirable properties at least approximately. Furthermore, different TF spectra that may be quite dissimilar theoretically yield approximately equal results for an underspread process. These claims will be substantiated mathematically by upper bounds on the respective approximation errors.

Our paper contains three further contributions.

- We show that all major TF spectra belong to one of two classes of spectra, termed type I and type II spectra. While the first class was previously studied in [6], [16], [19], [23], [24], the second class is new. Axiomatic definitions and compact formulations in terms of TF operator symbols are presented for both classes.

- We show that under certain conditions, the Gabor expansion [34], [35] provides an approximate decorrelation of an underspread process (approximate Karhunen-Loève 
(KL) transform), and TF spectra provide an approximate KL spectrum.

- We present approximate multiplicative input-output relations for the TF spectra of underspread processes that are passed through underspread linear time-varying (LTV) systems.

Taken together, our results show that the second-order analysis and linear filtering of underspread nonstationary processes are essentially as simple as for stationary processes. Our TF calculus provides a strong basis for applications in nonstationary statistical signal processing such as nonstationary signal estimation, detection, and coherence analysis [32], [36]-[50]. The resulting methods can be efficiently implemented using TF signal expansions like the Gabor transform or local cosine basis function expansions.

We note that parametric time-dependent spectra (e.g., [15], [51]-[53]), higher-order time-dependent spectra [54]-[58], and the estimation of TF spectra [14], [16], [19], [23], [24], [32], [59]-[68] are not considered here.

This paper is organized as follows. In Section II, we discuss the two classes of type I and type II spectra. General formulations of these classes in terms of TF operator symbols are given, and some important specific type I and type II spectra are considered. Section III reviews the fundamentals of underspread processes. In the five subsequent sections, the unique role of underspread processes in TF spectral analysis is demonstrated. It is shown that for underspread processes the various type I and type II spectra approximately satisfy many desirable properties (Sections IV and V), yield approximately equal results (Section VI), provide an approximate KL spectrum (Section VII), and permit the formulation of simple approximate input-output relations for linearly filtered processes (Section VIII). Finally, conclusions are provided in Section IX.

\section{TIME-FREQUENCY SPECTRA}

In this section, we present axiomatic definitions of type I and type II spectra and unified expressions of these spectra in terms of TF operator symbols. Our principal goal is to provide a mathematical basis for the underspread calculus to be established in Sections IV-VIII. Rather than advocating a specific spectrum, we will demonstrate in Sections IV-VIII that, for underspread processes, practically all spectra yield satisfactory and essentially equivalent results.

In what follows, the cross-correlation operator $\boldsymbol{R}_{x y}$ of two nonstationary random processes $x(t)$ and $y(t)$ is defined as the linear operator [69] whose kernel equals the cross-correlation function $r_{x y}\left(t_{1}, t_{2}\right)=\mathrm{E}\left\{x\left(t_{1}\right) y^{*}\left(t_{2}\right)\right\}$ (here, $\mathrm{E}$ and ${ }^{*}$ denote expectation and complex conjugation, respectively). The autocorrelation operator $\boldsymbol{R}_{x}$ of a single process $x(t)$ is defined as $\boldsymbol{R}_{x} \triangleq \boldsymbol{R}_{x x}$. All processes are assumed zero-mean.

\section{A. TF Operator Symbols}

Our formulation of type I and type II spectra will be based on TF symbols of LTV systems or linear operators. Consider a linear operator $\boldsymbol{H}$ with kernel $h\left(t_{1}, t_{2}\right)$ [69], [70]. A fairly wide class of linear TF representations of $H$ is given by the $T F$ shift covariant TF operator symbols ${ }^{1}$ [71]-[74]

$$
\begin{array}{r}
L_{\boldsymbol{H}}^{(\boldsymbol{P})}(t, f) \triangleq \int_{t_{1}} \int_{t_{2}} h\left(t_{1}, t_{2}\right) p^{*}\left(t_{1}-t, t_{2}-t\right) \\
\times e^{-j 2 \pi f\left(t_{1}-t_{2}\right)} d t_{1} d t_{2} .
\end{array}
$$

This class is parameterized by a "prototype operator" $\boldsymbol{P}$ with kernel $p\left(t_{1}, t_{2}\right)$. If $\boldsymbol{H}$ and $\boldsymbol{P}$ are Hilbert-Schmidt operators, the TF operator symbol can be rewritten as the inner product ${ }^{2}$

$$
L_{\boldsymbol{H}}^{(\boldsymbol{P})}(t, f)=\left\langle\boldsymbol{H}, \boldsymbol{P}_{t, f}\right\rangle, \quad \text { with } \boldsymbol{P}_{t, f}=\boldsymbol{S}_{t, f}^{(\alpha)} \boldsymbol{P} \boldsymbol{S}_{t, f}^{(\alpha)+} \text {. }
$$

Here, $S_{\tau, \nu}^{(\alpha)}$ is the generalized TF shift operator ${ }^{3}$ defined by

$$
\left(\boldsymbol{S}_{\tau, \nu}^{(\alpha)} x\right)(t)=x(t-\tau) e^{j 2 \pi \nu t} e^{j 2 \pi \tau \nu(\alpha-1 / 2)}, \quad \alpha \in \mathbb{R} .
$$

If $\boldsymbol{H}$ or $\boldsymbol{P}$ is not Hilbert-Schmidt, the integral representation (1) of $L_{\boldsymbol{H}}^{(\boldsymbol{P})}(t, f)$ may still be meaningful within a suitable framework of generalized functions; $\left\langle\boldsymbol{H}, \boldsymbol{P}_{t, f}\right\rangle$ will then be used merely as a compact notation for (1). An example of this case is the generalized Weyl symbol (GWS). The GWS of a linear operator $\boldsymbol{H}$ is defined as [75]-[83]

$$
L_{\boldsymbol{H}}^{(\alpha)}(t, f)=\int_{\tau} h^{(\alpha)}(t, \tau) e^{-j 2 \pi f \tau} d \tau
$$

with

$$
\begin{aligned}
h^{(\alpha)}(t, \tau) & =\left(\mathcal{M}_{\alpha} h\right)(t, \tau) \\
& \triangleq h\left(t+\left(\frac{1}{2}-\alpha\right) \tau, t-\left(\frac{1}{2}+\alpha\right) \tau\right)
\end{aligned}
$$

where $\alpha$ is a real-valued parameter. The GWS is a special case of the generic TF operator symbol (1) that is obtained for the nonHilbert-Schmidt prototype operator $\boldsymbol{P}$ with kernel $p\left(t_{1}, t_{2}\right)=$ $\delta\left(\left(\frac{1}{2}-\alpha\right) t_{1}+\left(\frac{1}{2}+\alpha\right) t_{2}\right)$. We note, however, that many of the bounds in Sections IV-VIII assume the Hilbert-Schmidt property to be satisfied by $\boldsymbol{H}$, where $\boldsymbol{H}=\boldsymbol{H}_{x}$ (an innovations system of the process $x(t)$, cf. Section II-C) or $\boldsymbol{H}=\boldsymbol{R}_{x}$ (the autocorrelation operator of $x(t)$ ).

Under certain conditions on $\boldsymbol{H}$ and $\boldsymbol{P}, L_{\boldsymbol{H}}^{(\boldsymbol{P})}(t, f)$ can be interpreted as a "TF transfer function" of $\boldsymbol{H}$ in the sense that it characterizes the amplification/attenuation caused by $\boldsymbol{H}$ about the TF point $(t, f)$ [81], [83]. Indeed, according to the inner

\footnotetext{
${ }^{1}$ All integrals are from $-\infty$ to $\infty$ unless stated otherwise.

${ }^{2}$ An operator $\boldsymbol{H}$ is Hilbert-Schmidt if it has a finite Hilbert-Schmidt operator norm$$
\|H\| \triangleq \sqrt{\operatorname{Tr}\left\{H H^{+}\right\}}<\infty
$$

with $\operatorname{Tr}\{\cdot\}$ and superscript + denoting the trace and adjoint of an operator, respectively [69], [70]. The inner product of two Hilbert-Schmidt operators is defined as

$$
\left\langle H_{1}, H_{2}\right\rangle \triangleq \operatorname{Tr}\left\{H_{1} H_{2}^{+}\right\}=\int_{t} \int_{t^{\prime}} h_{1}\left(t, t^{\prime}\right) h_{2}^{*}\left(t, t^{\prime}\right) d t d t^{\prime} .
$$

${ }^{3}$ The real-valued parameter $\alpha$ in $S_{\tau, \nu}^{(\alpha)}$ expresses a degree of freedom in defining a joint $\mathrm{TF}$ shift, which exists because time shifts and frequency shifts do not commute. For example, $\alpha=1 / 2(\alpha=-1 / 2)$ corresponds to performing the time shift before (after) the frequency shift. The operator $\boldsymbol{S}_{t, f}^{(\alpha)} \boldsymbol{P} \boldsymbol{S}_{t, f}^{(\alpha)+}$ does not depend on $\alpha$.
} 
product representation $\left\langle\boldsymbol{H}, \boldsymbol{P}_{t, f}\right\rangle, L_{\boldsymbol{H}}^{(\boldsymbol{P})}(t, f)$ measures the "strength" of $\boldsymbol{H}$ in the one-dimensional operator space spanned by $\boldsymbol{P}_{t, f}$. If $\boldsymbol{P}$ is a TF localization operator [45], [79], [84]-[87] about the origin of the TF plane, then $\boldsymbol{P}_{t, f}=\boldsymbol{S}_{t, f}^{(\alpha)} \boldsymbol{P} \boldsymbol{S}_{t, f}^{(\alpha)+}$ is associated with a neighborhood of the TF point $(t, f)$; the shape of this neighborhood is defined by $\boldsymbol{P}$.

\section{B. Type I Spectra}

The class of type I spectra has previously been studied in [6], [16], [23], [24] and, in a discrete-time setting, in [19]. The definition of type I spectra can be motivated by three properties of the PSD. Let $\tilde{r}_{x y}(\tau)=r_{x y}(t, t-\tau)=\mathrm{E}\left\{x(t) y^{*}(t-\tau)\right\}$ denote the cross-correlation function of two jointly wide-sense stationary processes $x(t)$ and $y(t)$. The cross-PSD of $x(t)$ and $y(t)$ is defined by the Wiener-Khintchine relation [88], [89]

$$
P_{x y}(f) \triangleq \int_{\tau} \tilde{r}_{x y}(\tau) e^{-j 2 \pi f \tau} d \tau .
$$

We note the following properties. First, the PSD depends linearly on $x(t)$ and semi-linearly on $y(t)$, i.e.,

$$
\begin{aligned}
x(t) & =a_{1} x_{1}(t)+a_{2} x_{2}(t) \\
& \Longrightarrow P_{x y}(f)=a_{1} P_{x_{1} y}(f)+a_{2} P_{x_{2} y}(f) \\
y(t) & =b_{1} y_{1}(t)+b_{2} y_{2}(t) \\
& \Longrightarrow P_{x y}(f)=b_{1}^{*} P_{x y_{1}}(f)+b_{2}^{*} P_{x y_{2}}(f) .
\end{aligned}
$$

Second, it is "covariant" to a frequency shift of both processes:

$$
\begin{aligned}
\tilde{x}(t) & =x(t) e^{j 2 \pi \nu t}, \quad \tilde{y}(t)=y(t) e^{j 2 \pi \nu t} \\
& \Longrightarrow P_{\tilde{x} \tilde{y}}(f)=P_{x y}(f-\nu) .
\end{aligned}
$$

Third, the auto-PSD $P_{x}(f) \triangleq P_{x x}(f) \geq 0$ integrates to the mean power, i.e.,

$$
\int_{f} P_{x}(f) d f=\mathrm{E}\left\{|x(t)|^{2}\right\}
$$

These properties can be extended to nonstationary processes $x(t), y(t)$ as follows [6], [16], [24].

Definition 1: The class of type I TF spectra $P_{x y}^{(\mathrm{I})}(t, f)$ is defined by the following axioms.

1) Superposition property:

$$
\begin{aligned}
x(t) & =a_{1} x_{1}(t)+a_{2} x_{2}(t) \\
& \Longrightarrow P_{x y}^{(\mathrm{I})}(t, f)=a_{1} P_{x_{1} y}^{(\mathrm{I})}(t, f)+a_{2} P_{x_{2} y}^{(\mathrm{I})}(t, f) \\
y(t) & =b_{1} y_{1}(t)+b_{2} y_{2}(t) \\
& \Longrightarrow P_{x y}^{(\mathrm{I})}(t, f)=b_{1}^{*} P_{x y_{1}}^{(\mathrm{I})}(t, f)+b_{2}^{*} P_{x y_{2}}^{(\mathrm{I})}(t, f) .
\end{aligned}
$$

2) TF shift covariance:

$$
\begin{aligned}
\tilde{x}(t) & =\left(\boldsymbol{S}_{\tau, \nu}^{(\alpha)} x\right)(t), \quad \tilde{y}(t)=\left(\boldsymbol{S}_{\tau, \nu}^{(\alpha)} y\right)(t) \\
& \Longrightarrow P_{\tilde{x} \tilde{y}}^{(\mathrm{I})}(t, f)=P_{x y}^{(\mathrm{I})}(t-\tau, f-\nu) .
\end{aligned}
$$

3) TF energy distribution property: for any process $x(t)$ with finite mean energy ${ }^{4} \bar{E}_{x} \triangleq \mathrm{E}\left\{\|x\|_{2}^{2}\right\}=\operatorname{Tr}\left\{\boldsymbol{R}_{x}\right\}$

$$
\int_{t} \int_{f} P_{x x}^{(\mathrm{I})}(t, f) d t d f=\bar{E}_{x} .
$$

The following theorem ${ }^{5}$ establishes an explicit characterization of the class of type I spectra (cf. [6], [16], [24]).

Theorem 2: Any type I spectrum can be written as

$$
P_{x y}^{(\mathrm{I})}(t, f)=L_{\boldsymbol{R}_{x y}}^{(\boldsymbol{P})}(t, f)=\left\langle\boldsymbol{R}_{x y}, \boldsymbol{P}_{t, f}\right\rangle
$$

where $\boldsymbol{P}$ is an arbitrary trace-normalized linear operator (i.e., $\operatorname{Tr}\{\boldsymbol{P}\}=1$ ) and $\boldsymbol{P}_{t, f}$ is defined in (2). Conversely, any spectrum of the form (11) with a trace-normalized $\boldsymbol{P}$ is a type I spectrum.

Hence, we see that the class of type I spectra is identical to the class of TF operator symbols (1) of the correlation operator $R_{x y}$ with a trace-normalized prototype operator $\boldsymbol{P}$. It can furthermore be shown that under weak conditions

$$
P_{x y}^{(\mathrm{I})}(t, f)=\mathrm{E}\left\{\left\langle x, \boldsymbol{P}_{t, f} y\right\rangle\right\} .
$$

Here, $\left\langle x, \boldsymbol{P}_{t, f} y\right\rangle$ is a member of Cohen's class of bilinear TF signal representations [24], [90], [91]. Hence, type I spectra can also be viewed as the expectation of corresponding TF signal representations from Cohen's class. If $\boldsymbol{P}$ is a TF localization operator [45], [79], [84]-[87] about the origin of the TF plane, $P_{t, f}$ is a TF localization operator about the TF point $(t, f)$. Consequently, the auto-spectrum

$$
P_{x}^{(\mathrm{I})}(t, f) \triangleq P_{x x}^{(\mathrm{I})}(t, f)=\mathrm{E}\left\{\left\langle x, \boldsymbol{P}_{t, f} x\right\rangle\right\}
$$

can be interpreted as the mean energy of the process $x(t)$ about the TF point $(t, f)$.

There exist four "canonical" formulations of type I spectra that are based on representations of the operators $\boldsymbol{P}$ and $\boldsymbol{R}_{x y}$ in four different domains (all related by Fourier transforms):

$$
\begin{aligned}
& P_{x y}^{(\mathrm{I})}(t, f) \\
& =\int_{t_{1}} \int_{t_{2}} r_{x y}\left(t_{1}, t_{2}\right) p^{*}\left(t_{1}-t, t_{2}-t\right) e^{-j 2 \pi f\left(t_{1}-t_{2}\right)} d t_{1} d t_{2} \\
& =\int_{f_{1}} \int_{f_{2}} r_{X Y}\left(f_{1}, f_{2}\right) B_{\boldsymbol{P}}^{*}\left(f_{1}-f, f_{2}-f\right) e^{j 2 \pi t\left(f_{1}-f_{2}\right)} d f_{1} d f_{2} \\
& =\int_{t^{\prime}} \int_{f^{\prime}} \bar{W}_{x y}^{(\alpha)}\left(t^{\prime}, f^{\prime}\right) L_{\boldsymbol{P}}^{(\alpha) *}\left(t^{\prime}-t, f^{\prime}-f\right) d t^{\prime} d f^{\prime} \\
& =\int_{\tau} \int_{\nu} \bar{A}_{x y}^{(\alpha)}(\tau, \nu) S_{\boldsymbol{P}}^{(\alpha) *}(\tau, \nu) e^{j 2 \pi(t \nu-f \tau)} d \tau d \nu .
\end{aligned}
$$

${ }^{4}$ Throughout the paper, $\|f\|_{p}$ denotes the $L_{p}\left(\mathbb{R}^{n}\right) \operatorname{norm}(n=1,2)$, i.e.,

$$
\begin{aligned}
& \|f\|_{p} \triangleq\left[\int \ldots \int\left|f\left(x_{1}, \ldots, x_{n}\right)\right|^{p} d x_{1} \ldots d x_{n}\right]^{1 / p}, 1 \leq p<\infty \\
& \|f\|_{\infty} \triangleq \sup \left|f\left(x_{1}, \ldots, x_{n}\right)\right| .
\end{aligned}
$$

and

Furthermore, the $L_{2}\left(\mathbb{R}^{n}\right)$ inner product is defined as

$$
\langle f, g\rangle \triangleq \int \ldots \int f\left(x_{1}, \ldots, x_{n}\right) g^{*}\left(x_{1}, \ldots, x_{n}\right) d x_{1} \ldots d x_{n} .
$$

${ }^{5}$ Proofs of this and subsequent theorems can be found in [30]. 
Here, $\boldsymbol{P}$ is represented by its kernel $p\left(t_{1}, t_{2}\right)$, its bi-frequency function [92]

$$
B_{\boldsymbol{P}}\left(f_{1}, f_{2}\right) \triangleq \int_{t_{1}} \int_{t_{2}} p\left(t_{1}, t_{2}\right) e^{-j 2 \pi\left(f_{1} t_{1}-f_{2} t_{2}\right)} d t_{1} d t_{2}
$$

its GWS $L_{P}^{(\alpha)}(t, f)$ (cf. (4)), and its generalized spreading function (GSF) $S_{\boldsymbol{P}}^{(\alpha)}(\tau, \nu)$. The GSF is defined by [78], [93]

$$
S_{\boldsymbol{P}}^{(\alpha)}(\tau, \nu) \triangleq \int_{t} p^{(\alpha)}(t, \tau) e^{-j 2 \pi \nu t} d t
$$

with $p^{(\alpha)}(t, \tau)=\left(\mathcal{M}_{\alpha} p\right)(t, \tau)$ (cf. (5)). Similarly, $\boldsymbol{R}_{x y}$ is represented by the correlation function $r_{x y}\left(t_{1}, t_{2}\right)$, the frequency-domain correlation function

$$
r_{X Y}\left(f_{1}, f_{2}\right)=\mathrm{E}\left\{X\left(f_{1}\right) Y^{*}\left(f_{2}\right)\right\},
$$

the generalized Wigner-Ville spectrum

$$
\bar{W}_{x y}^{(\alpha)}(t, f)=L_{\boldsymbol{R}_{x y}}^{(\alpha)}(t, f)
$$

(see Section II-D), and the generalized expected ambiguity function

$$
\bar{A}_{x y}^{(\alpha)}(\tau, \nu)=S_{\boldsymbol{R}_{x y}}^{(\alpha)}(\tau, \nu)
$$

(see Section III-A). We note that the expressions (12a) and (12b) are valid for arbitrary $\alpha \in \mathbb{R}$; their result, $P_{x y}^{(\mathrm{I})}(t, f)$, does not depend on $\alpha$. In what follows, we will restrict to type I autospectra for simplicity. For more details on type I cross-spectra see [48], [94].

\section{Type II Spectra}

The definition of the new class of type II spectra is motivated by the relation between PSD and innovations systems in the stationary case. The innovations system representation of a (generally nonstationary) random process $x(t)$ is given by [11], [22], [89], [95]

$$
x(t)=\left(\boldsymbol{H}_{x} n\right)(t)=\int_{t^{\prime}} h_{x}\left(t, t^{\prime}\right) n\left(t^{\prime}\right) d t^{\prime} .
$$

Here, $n(t)$ denotes normalized stationary white noise (with correlation operator $\boldsymbol{R}_{n}=\boldsymbol{I}$ ) and $\boldsymbol{H}_{x}$ is an innovations system for the process $x(t)$. All innovations systems for $x(t)$ are solutions of the operator equation $\boldsymbol{H}_{x} \boldsymbol{H}_{x}^{+}=\boldsymbol{R}_{x}$. Note that if $\boldsymbol{H}_{x}$ is an innovations system for $x(t)$, then so is $\tilde{\boldsymbol{H}}_{x}=\boldsymbol{H}_{x} \boldsymbol{U}$ with an arbitrary operator $\boldsymbol{U}$ satisfying $\boldsymbol{U} \boldsymbol{U}^{+}=\boldsymbol{I}$.

For a stationary process $x(t)$, the PSD can be written as

$$
P_{x}(f)=\left|H_{x}(f)\right|^{2}
$$

where $H_{x}(f)$ is the transfer function of an arbitrary time-invariant innovations system $\boldsymbol{H}_{x}$ for $x(t)$. As a system representation, the transfer function has three important properties: it is linear, covariant to frequency shifts of the system, and normalized in that $H(f) \equiv 1$ for the identity system $\boldsymbol{H}=\boldsymbol{I}$. By a natural extension to the nonstationary case, we define a type II spectrum as the squared magnitude of a linear, TF shift covariant, and normalized "TF transfer function" of an LTV innovations system $\boldsymbol{H}_{x}$.

Definition 3: The class of type II TF spectra $P_{x}^{(\mathrm{II})}(t, f)$ is defined by

$$
P_{x}^{(\mathrm{II})}(t, f)=\left|D_{\boldsymbol{H}_{x}}(t, f)\right|^{2}
$$

where $\boldsymbol{H}_{x}$ is an innovations system for $x(t)$ and the TF operator representation $D_{\boldsymbol{H}}(t, f)$ is required to satisfy the following axioms.

1) Linearity: $\boldsymbol{H}=a_{1} \boldsymbol{H}_{1}+a_{2} \boldsymbol{H}_{2}$

$$
\Longrightarrow D_{\boldsymbol{H}}(t, f)=a_{1} D_{\boldsymbol{H}_{1}}(t, f)+a_{2} D_{\boldsymbol{H}_{2}}(t, f) \text {. }
$$

2) TF shift covariance: ${ }^{6} \tilde{\boldsymbol{H}}=\boldsymbol{S}_{\tau, \nu}^{(\alpha)} \boldsymbol{H} \boldsymbol{S}_{\tau, \nu}^{(\alpha)+}$

$$
\Longrightarrow D_{\tilde{\boldsymbol{H}}}(t, f)=D_{\boldsymbol{H}}(t-\tau, f-\nu) \text {. }
$$

3) Normalization: $\boldsymbol{H}=\boldsymbol{I} \Longrightarrow D_{\boldsymbol{H}}(t, f) \equiv 1$.

The next theorem states that the TF transfer function $D_{\boldsymbol{H}}(t, f)$ must be chosen as a TF shift covariant TF operator symbol $L_{\boldsymbol{H}}^{(\boldsymbol{P})}(t, f)$ as defined in (1).

Theorem 4: Any type II spectrum can be written as

$$
P_{x}^{(\mathrm{II})}(t, f)=\left|L_{\boldsymbol{H}_{x}}^{(\boldsymbol{P})}(t, f)\right|^{2}=\left|\left\langle\boldsymbol{H}_{x}, \boldsymbol{P}_{t, f}\right\rangle\right|^{2}
$$

where $\boldsymbol{P}$ is an arbitrary trace-normalized linear operator (i.e., $\operatorname{Tr}\{\boldsymbol{P}\}=1$ ). Conversely, any spectrum of the form (14) with a trace-normalized $\boldsymbol{P}$ is a type II spectrum.

It is seen that for a given process $x(t)$ (i.e., for a given correlation $\left.\boldsymbol{R}_{x}\right), P_{x}^{(\mathrm{II})}(t, f)$ depends on the choice of i) the prototype operator $\boldsymbol{P}$ and ii) the innovations system $\boldsymbol{H}_{x}$. Note that the latter choice corresponds to an ambiguity that does not exist in the case of type I spectra.

By comparing (14) and (11), we realize that type II spectra equal the squared magnitude of a TF transfer function of an innovations system $\boldsymbol{H}_{x}$ while type I spectra equal a TF transfer function of the "squared" innovations system $\boldsymbol{H}_{x} \boldsymbol{H}_{x}^{+}=\boldsymbol{R}_{x}$. Whereas in the stationary case these two approaches both yield the PSD, in the nonstationary case they result in two different classes of TF spectra.

Similarly to type I spectra, type II spectra admit canonical formulations in terms of representations of the operators $\boldsymbol{H}_{x}$ and $\boldsymbol{P}$ in four different domains that are related by Fourier transforms:

$$
\begin{aligned}
P_{x}^{(\mathrm{II})}(t, f) & \mid \int_{t_{1}} \int_{t_{2}} h_{x}\left(t_{1}, t_{2}\right) p^{*}\left(t_{1}-t, t_{2}-t\right) \\
& \times\left. e^{-j 2 \pi f\left(t_{1}-t_{2}\right)} d t_{1} d t_{2}\right|^{2} \\
= & \mid \int_{f_{1}} \int_{f_{2}} B_{\boldsymbol{H}_{x}}\left(f_{1}, f_{2}\right) B_{\boldsymbol{P}}^{*}\left(f_{1}-f, f_{2}-f\right) \\
& \times\left. e^{j 2 \pi t\left(f_{1}-f_{2}\right)} d f_{1} d f_{2}\right|^{2} \\
= & \left|\int_{t^{\prime}} \int_{f^{\prime}} L_{\boldsymbol{H}_{x}}^{(\alpha)}\left(t^{\prime}, f^{\prime}\right) L_{\boldsymbol{P}}^{(\alpha) *}\left(t^{\prime}-t, f^{\prime}-f\right) d t^{\prime} d f^{\prime}\right|^{2} \\
= & \left|\int_{\tau} \int_{\nu} S_{\boldsymbol{H}_{x}}^{(\alpha)}(\tau, \nu) S_{\boldsymbol{P}}^{(\alpha) *}(\tau, \nu) e^{j 2 \pi(t \nu-f \tau)} d \tau d \nu\right|^{2} .
\end{aligned}
$$

\section{Special Cases}

Virtually all nonparametric time-varying spectra proposed so far in the literature fit within the classes of type I and type II

\footnotetext{
${ }^{6}$ We note that if $\boldsymbol{H}_{x}$ is an innovations system for $x(t)$, then the TF shifted operator $\tilde{H}_{x}=\boldsymbol{S}_{\tau, \nu}^{(\alpha)} \boldsymbol{H}_{x} \boldsymbol{S}_{\tau, \nu}^{(\alpha)+}$ is an innovations system for the TF shifted process $\tilde{x}(t)=\left(\boldsymbol{S}_{\tau, \nu}^{\left(\alpha^{\prime}\right)} x\right)(t)\left(\alpha^{\prime} \neq \alpha\right.$ allowed $)$.
} 
spectra. To support this claim and for later reference, we next consider some important special cases of type I and type II spectra within our TF operator symbol framework. Some of these TF spectra are new. Spectra of either class using the same prototype operator $\boldsymbol{P}$ will be discussed jointly.

1) Generalized Wigner-Ville Spectrum and Generalized Evolutionary Spectrum: The generalized Wigner-Ville spectrum (GWVS) is defined as [16], [23], [24]

$$
\bar{W}_{x}^{(\alpha)}(t, f) \triangleq \int_{\tau} r_{x}^{(\alpha)}(t, \tau) e^{-j 2 \pi f \tau} d \tau
$$

with $r_{x}^{(\alpha)}(t, \tau)=\left(\mathcal{M}_{\alpha} r_{x}\right)(t, \tau)$. The GWVS is a family of type I spectra whose underlying TF operator symbol is the GWS $L_{\boldsymbol{H}}^{(\alpha)}(t, f)$ in (4), i.e., $\bar{W}_{x}^{(\alpha)}(t, f)=L_{\boldsymbol{R}_{x}}^{(\alpha)}(t, f)$. Two important members of the GWVS family are the Wigner-Ville spectrum [4], [8], [14], [23], [24], [27], [29], [30], [94], [96]-[98] and the Rihaczek spectrum [7], [99], which are obtained for $\alpha=0$ and $\alpha=1 / 2$, respectively. The GWVS can also be written as

$$
\bar{W}_{x}^{(\alpha)}(t, f)=\int_{\tau} \int_{\nu} \bar{A}_{x}^{(\alpha)}(\tau, \nu) e^{-j 2 \pi(f \tau-t \nu)} d \tau d \nu
$$

where $\bar{A}_{x}^{(\alpha)}(\tau, \nu)$ denotes the generalized expected ambiguity function to be defined in Section III-A. Since $\bar{A}_{x}^{(\alpha)}(\tau, \nu)$ can be interpreted as a TF correlation function, (16) provides an extension of the Wiener-Khintchine relation (6) to the nonstationary case. Applications of the GWVS in nonstationary statistical signal processing were described in [32], [37], [42], [44], [46], [47], [50].

The type II counterpart of the GWVS is the generalized evolutionary spectrum (GES) [22], [29], [30]

$$
G_{x}^{(\alpha)}(t, f)=\left|\int_{\tau} h_{x}^{(\alpha)}(t, \tau) e^{-j 2 \pi f \tau} d \tau\right|^{2} .
$$

Again, the underlying TF operator symbol is the GWS $L_{\boldsymbol{H}}^{(\alpha)}(t, f)$, i.e., $G_{x}^{(\alpha)}(t, f)=\left|L_{\boldsymbol{H}_{x}}^{(\alpha)}(t, f)\right|^{2}$. The evolutionary spectrum [5], [100], the transitory evolutionary spectrum [20], [22], and the Weyl spectrum [22] are important special cases of the GES obtained with $\alpha=1 / 2, \alpha=-1 / 2$, and $\alpha=0$, respectively.

2) Type I and Type II Page Spectrum: Page defined the instantaneous power spectrum [1], [2], [13], [17]

$$
P_{x}^{(\mathrm{I})}(t, f)=\mathrm{E}\left\{\frac{d}{d t}\left|X_{t}(f)\right|^{2}\right\}
$$

with $X_{t}(f) \triangleq \int_{-\infty}^{t} x(\tau) e^{-j 2 \pi f \tau} d \tau$. This spectrum can be rewritten as

$$
P_{x}^{(\mathrm{I})}(t, f)=2 \Re\left\{\int_{0}^{\infty} r_{x}(t, t-\tau) e^{-j 2 \pi f \tau} d \tau\right\} .
$$

It belongs to the class of type I spectra and will here be referred to as type I Page spectrum. The underlying TF operator symbol is the "Page symbol" [74]

$$
L_{\boldsymbol{H}}^{(\boldsymbol{P})}(t, f)=2 \Re\left\{\int_{0}^{\infty} h(t, t-\tau) e^{-j 2 \pi f \tau} d \tau\right\} .
$$

The type I Page spectrum satisfies the property of causality. As the corresponding type II spectrum, we define the new type II
Page spectrum by using the Page symbol as the TF operator symbol in (14), i.e.,

$$
P_{x}^{(\mathrm{II})}(t, f)=4\left[\Re\left\{\int_{0}^{\infty} h_{x}(t, t-\tau) e^{-j 2 \pi f \tau} d \tau\right\}\right]^{2} .
$$

3) Type I and Type II Levin Spectrum: Levin [3] augmented Page's definition by adding an anticausal counterpart to (18). He arrived at the (type I) Levin spectrum

$$
P_{x}^{(\mathrm{I})}(t, f)=\Re\left\{\int_{\tau} r_{x}(t, t-\tau) e^{-j 2 \pi f \tau} d \tau\right\}
$$

which is equal to the real part of the Rihaczek spectrum $\bar{W}_{x}^{(1 / 2)}(t, f)$. The underlying TF operator symbol is the "Levin symbol" [74]

$$
L_{\boldsymbol{H}}^{(\boldsymbol{P})}(t, f)=\Re\left\{\int_{\tau} h(t, t-\tau) e^{-j 2 \pi f \tau} d \tau\right\} .
$$

Using the same TF operator symbol in (14) yields the new type II Levin spectrum

$$
P_{x}^{(\mathrm{II})}(t, f)=\left[\Re\left\{\int_{\tau} h_{x}(t, t-\tau) e^{-j 2 \pi f \tau} d \tau\right\}\right]^{2} .
$$

4) Type I and Type II Physical Spectrum: Motivated by the idea of measuring the mean energy about a TF analysis point via an inner product, the (type I) physical spectrum was defined as the expectation of the spectrogram of $x(t)$ [8], [101]

$$
\mathrm{PS}_{x}^{(g)}(t, f) \triangleq \mathrm{E}\left\{\left|\left\langle x, g_{t, f}\right\rangle\right|^{2}\right\} .
$$

Here, $g_{t, f}\left(t^{\prime}\right) \triangleq\left(\boldsymbol{S}_{t, f}^{(\alpha)} g\right)\left(t^{\prime}\right)$, where $g(t)$ is an analysis window localized about the origin of the TF plane and normalized such that $\|g\|_{2}=1$. The type I physical spectrum can be rewritten as

$$
\operatorname{PS}_{x}^{(g)}(t, f)=\left\langle\boldsymbol{R}_{x} g_{t, f}, g_{t, f}\right\rangle=\left\langle\boldsymbol{R}_{x},\left(g \otimes g^{*}\right)_{t, f}\right\rangle .
$$

The prototype operator $\boldsymbol{P}$ is the rank-one TF localization operator $g \otimes g^{*}$ with kernel $p\left(t_{1}, t_{2}\right)=g\left(t_{1}\right) g^{*}\left(t_{2}\right)$. The corresponding TF operator symbol

$$
L_{\boldsymbol{H}}^{(\boldsymbol{P})}(t, f)=L_{\boldsymbol{H}}^{\left(g \otimes g^{*}\right)}(t, f)=\left\langle\boldsymbol{H},\left(g \otimes g^{*}\right)_{t, f}\right\rangle=\left\langle\boldsymbol{H} g_{t, f}, g_{t, f}\right\rangle
$$

is known as the lower symbol [27], [30], [76]. Using the lower symbol in (14), we obtain the new type II physical spectrum

$$
P_{x}^{(\mathrm{II})}(t, f)=\left|L_{\boldsymbol{H}_{x}}^{\left(g \otimes g^{*}\right)}(t, f)\right|^{2}=\left|\left\langle\boldsymbol{H}_{x},\left(g \otimes g^{*}\right)_{t, f}\right\rangle\right|^{2} .
$$

5) Type I and Type II Multiwindow Physical Spectrum: The type I multiwindow physical spectrum (cf. [19], [27]) is defined as the linear combination of several type I physical spectra with linearly independent, normalized analysis windows $g_{k}(t)$

$$
P_{x}^{(\mathrm{I})}(t, f)=\sum_{k=1}^{N} \gamma_{k} \operatorname{PS}_{x}^{\left(g_{k}\right)}(t, f)=\sum_{k=1}^{N} \gamma_{k}\left\langle\boldsymbol{R}_{x},\left(g_{k} \otimes g_{k}^{*}\right)_{t, f}\right\rangle .
$$

Here, the coefficients $\gamma_{k}$ are assumed to be normalized such that $\sum_{k=1}^{N} \gamma_{k}=1$. The prototype operator is $\boldsymbol{P}=\sum_{k=1}^{N} \gamma_{k}^{*} g_{k} \otimes g_{k}^{*}$; the TF operator symbol is

$$
L_{\boldsymbol{H}}^{(\boldsymbol{P})}(t, f)=\sum_{k=1}^{N} \gamma_{k}\left\langle\boldsymbol{H},\left(g_{k} \otimes g_{k}^{*}\right)_{t, f}\right\rangle .
$$

Any type I spectrum with a finite-rank, normal, trace-normalized prototype operator is a multiwindow physical spectrum. Multiwindow physical spectra may be useful as finite-rank approximations to general type I spectra (cf. [19]). Using the same 
prototype operator in (14), we obtain the new type II multiwindow physical spectrum

$$
P_{x}^{(\mathrm{II})}(t, f)=\left|\sum_{k=1}^{N} \gamma_{k} L_{\boldsymbol{H}_{x}}^{\left(g_{k} \otimes g_{k}^{*}\right)}(t, f)\right|^{2} .
$$

\section{UNDERSPREAD NONSTATIONARY PROCESSES}

The main goal of this paper is to describe the privileged role that underspread processes play in nonstationary spectral analysis. In this section, we explain the underspread property and introduce a concept of underspread processes that extends the original definition given by Kozek [25]-[28], [60]. We first review a TF correlation function on which the underspread concept is based.

\section{A. The Generalized Expected Ambiguity Function}

Processes are often characterized in terms of their correlation structure-for example, a stationary processes has temporal correlations but no spectral correlations, whereas a nonstationary white process has spectral correlations but no temporal correlations. A joint description of the temporal and spectral correlations is provided by the generalized expected ambiguity function (GEAF) defined as [25]-[27], [30]

$$
\bar{A}_{x}^{(\alpha)}(\tau, \nu) \triangleq \mathrm{E}\left\{\left\langle x, \boldsymbol{S}_{\tau, \nu}^{(\alpha)} x\right\rangle\right\}=\int_{t} r_{x}^{(\alpha)}(t, \tau) e^{-j 2 \pi \nu t} d t .
$$

Comparing this definition with the GSF in (13), it is seen that the GEAF is the GSF of the correlation operator $\boldsymbol{R}_{x}$, i.e., $\bar{A}_{x}^{(\alpha)}(\tau, \nu)=S_{\boldsymbol{R}_{x}}^{(\alpha)}(\tau, \nu)$.

The GEAF is a global measure of the correlation of all components of $x(t)$ that are separated by $\tau$ in time and by $\nu$ in frequency [25]-[27], [30]. For example, the GEAF of a stationary white process with PSD $\eta$ is $\bar{A}_{x}^{(\alpha)}(\tau, \nu)=\eta \delta(\tau) \delta(\nu)$. Thus, $\bar{A}_{x}^{(\alpha)}(\tau, \nu)=0$ unless $\tau$ and $\nu$ are both zero, which correctly indicates that the process has neither temporal nor spectral correlations. For a stationary process with correlation function $r_{x}\left(t_{1}, t_{2}\right)=\tilde{r}_{x}\left(t_{1}-t_{2}\right)$, we obtain $\bar{A}_{x}^{(\alpha)}(\tau, \nu)=\tilde{r}_{x}(\tau) \delta(\nu)$, which indicates the absence of spectral correlations. For a nonstationary white process with correlation function $r_{x}\left(t_{1}, t_{2}\right)=$ $q_{x}\left(t_{1}\right) \delta\left(t_{1}-t_{2}\right)$, we have $\bar{A}_{x}^{(\alpha)}(\tau, \nu)=Q_{x}(\nu) \delta(\tau)$ (where $Q_{x}(\nu)$ is the Fourier transform of $\left.q_{x}(t)\right)$, which indicates the absence of temporal correlations. The relation

$$
\bar{A}_{x}^{\left(\alpha_{2}\right)}(\tau, \nu)=\bar{A}_{x}^{\left(\alpha_{1}\right)}(\tau, \nu) e^{j 2 \pi\left(\alpha_{1}-\alpha_{2}\right) \tau \nu}
$$

allows us to use the shorthand notation

$$
\left|\bar{A}_{x}(\tau, \nu)\right| \triangleq\left|\bar{A}_{x}^{(\alpha)}(\tau, \nu)\right| \text {. }
$$

Further properties of the GEAF are described in [25], [27], [30].

\section{B. Underspread Processes}

The extension of the GEAF $\bar{A}_{x}^{(\alpha)}(\tau, \nu)$ in the $\tau$ and $\nu$ direction describes, respectively, the temporal and spectral correlation width of the process $x(t)$. A process that has noticeable correlations only for small TF lags $(\tau, \nu)$ will be called underspread. The GEAF of an underspread process is concentrated about the origin of the $(\tau, \nu)$ plane. In contrast, processes that have strong correlations for large TF lags $(\tau, \nu)$ will be termed overspread; their GEAF is not concentrated about the origin. Many nonstationary processes encountered in practical applications are underspread.

Underspread processes were first defined by Kozek [25]-[27] as those processes whose GEAF is exactly zero outside a compact support region $\mathcal{G}_{x}$ about the origin with area much less than one. More specifically, the underspread property was defined as $\sigma_{x} \ll 1$, with the correlation spread defined by

$$
\sigma_{x} \triangleq 4 \tau_{x}^{(\max )} \nu_{x}^{(\max )}
$$

where

$$
\tau_{x}^{(\max )} \triangleq \max _{(\tau, \nu) \in \mathcal{G}_{x}}|\tau|, \quad \nu_{x}^{(\max )} \triangleq \max _{(\tau, \nu) \in \mathcal{G}_{x}}|\nu| .
$$

1) Extended Underspread Concept: While the above definition of underspread processes is very simple, its usefulness is limited because practical processes rarely have compactly supported GEAFs. Using an effective GEAF support instead-i.e., approximating a noncompactly supported GEAF by a compactly supported one-is problematic since the modeling errors incurred by a specific choice of such an effective support are difficult to quantify. Therefore, we now propose an extended concept of underspread processes that uses as global measures of the "joint TF correlation width" the following weighted integrals of the GEAF [30]:

$$
\begin{aligned}
m_{x}^{(\phi)} & \triangleq \frac{\int_{\tau} \int_{\nu} \phi(\tau, \nu)\left|\bar{A}_{x}(\tau, \nu)\right| d \tau d \nu}{\int_{\tau} \int_{\nu}\left|\bar{A}_{x}(\tau, \nu)\right| d \tau d \nu} \\
& =\frac{1}{\left\|\bar{A}_{x}\right\|_{1}} \int_{\tau} \int_{\nu} \phi(\tau, \nu)\left|\bar{A}_{x}(\tau, \nu)\right| d \tau d \nu \\
M_{x}^{(\phi)} & \triangleq\left[\frac{\int_{\tau} \int_{\nu} \phi^{2}(\tau, \nu)\left|\bar{A}_{x}(\tau, \nu)\right|^{2} d \tau d \nu}{\int_{\tau} \int_{\nu}\left|\bar{A}_{x}(\tau, \nu)\right|^{2} d \tau d \nu}\right]^{1 / 2} \\
& =\frac{1}{\left\|\bar{A}_{x}\right\|_{2}}\left[\int_{\tau} \int_{\nu} \phi^{2}(\tau, \nu)\left|\bar{A}_{x}(\tau, \nu)\right|^{2} d \tau d \nu\right]^{1 / 2} .
\end{aligned}
$$

Here, $\phi(\tau, \nu)$ is a nonnegative weighting function that satisfies $\phi(0,0)=0$ and penalizes off-origin GEAF contributions. Special cases of these weighted integrals are the GEAF moments $m_{x}^{(k, l)} \triangleq m_{x}^{\left(\phi_{k}, l\right)}$ and $M_{x}^{(k, l)} \triangleq M_{x}^{\left(\phi_{k}, l\right)}$ obtained with the weighting functions $\phi_{k, l}(\tau, \nu)=|\tau|^{k}|\nu|^{l}[30]$. Note that $m_{x}^{(k, 0)}$ and $M_{x}^{(k, 0)}$ measure the temporal correlation width whereas $m_{x}^{(0, l)}$ and $M_{x}^{(0, l)}$ measure the spectral correlation width.

We now call a nonstationary process $x(t)$ underspread if suitable GEAF integrals $m_{x}^{(\phi)}$ and $M_{x}^{(\phi)}$ or GEAF moments $m_{x}^{(k, l)}$ and $M_{x}^{(k, l)}$ are "small." This extended concept of underspread processes does not require that the GEAF has compact support, and thus, it corresponds to a larger and more practically relevant class of processes. It is, however, less simple than the previous definition based on a compact GEAF support. Fig. 1 depicts schematically the GEAF of some example processes that are underspread in this extended sense.

The difference between the two underspread concepts is somewhat analogous to the difference between exact band limitation and effective band limitation of a signal, and our GEAF integrals and moments are analogous to various measures used to quantify the effective band limitation of a signal. In fact, placing constraints on different GEAF integrals or moments (e.g., requiring that $m_{x}^{(1,1)} \ll 1$ or $M_{x}^{(2,1)} \ll 1$ ) corresponds 


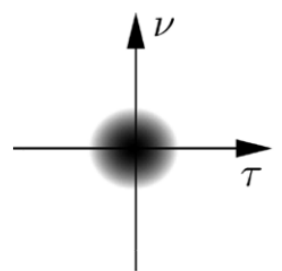

(a)

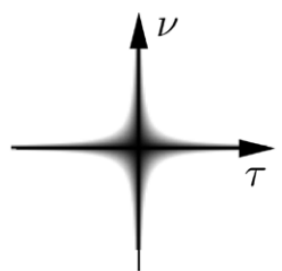

(b)

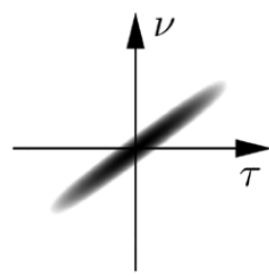

(c)
Fig. 1. Schematic representation of the GEAF magnitude of (a) an underspread process with small $m_{x}^{(k, 0)} m_{x}^{(0, l)}$ and $M_{x}^{(k, 0)} M_{x}^{(0, l)}$, (b) an underspread process with small $m_{x}^{(k, k)}$ and $M_{x}^{(k, k)}$, (c) a "chirpy" underspread process with small $m_{x}^{(\phi)}$ and $M_{x}^{(\phi)}$, where $\phi(\tau, \nu)$ has skew orientation.

to different definitions of the underspread property. Each one of these different definitions is mathematically precise, but no single definition works equally well in all applications. The many alternative ways of expressing the underspread concept mathematically provide a flexibility of mathematical analysis that will be exploited in Sections IV-VIII.

The "maximally underspread" process is stationary white noise, which has no temporal or spectral correlations whatsoever. The underspread property is not equivalent to the property of quasi-stationarity. Indeed, a quasi-stationary process may be overspread if its effective temporal correlation width is large; conversely, a highly nonstationary process may be underspread if its effective temporal correlation is short enough.

2) Innovations System Based Underspread Concept: An alternative view of an underspread process $x(t)$ is provided by the GSF of an innovations system $\boldsymbol{H}_{x}$ for $x(t), S_{\boldsymbol{H}_{x}}^{(\alpha)}(\tau, \nu)$. We call an innovations system $\boldsymbol{H}_{x}$ underspread if the TF shifts it produces are only by small time lags (delays) $\tau$ and small frequency (Doppler) lags $\nu$ [27], [30], [81], [83]. The TF shifts of $\boldsymbol{H}_{x}$ are described by its GSF $S_{\boldsymbol{H}_{x}}^{(\alpha)}(\tau, \nu)$. Therefore, $\boldsymbol{H}_{x}$ is underspread if and only if $S_{\boldsymbol{H}_{x}}^{(\alpha)}(\tau, \nu)$ is concentrated about the origin of the $(\tau, \nu)$ plane. The concentration of ${ }^{7}\left|S_{\boldsymbol{H}_{x}}(\tau, \nu)\right|$ about the origin can be measured by the weighted GSF integrals [30], [83]

$$
\begin{aligned}
m_{\boldsymbol{H}_{x}}^{(\phi)} & \triangleq \frac{\int_{\tau} \int_{\nu} \phi(\tau, \nu)\left|S_{\boldsymbol{H}_{x}}(\tau, \nu)\right| d \tau d \nu}{\int_{\tau} \int_{\nu}\left|S_{\boldsymbol{H}_{x}}(\tau, \nu)\right| d \tau d \nu} \\
& =\frac{1}{\| S_{\boldsymbol{H}_{x} \|_{1}}} \int_{\tau} \int_{\nu} \phi(\tau, \nu)\left|S_{\boldsymbol{H}_{x}}(\tau, \nu)\right| d \tau d \nu \\
M_{\boldsymbol{H}_{x}}^{(\phi)} & \triangleq\left[\frac{\int_{\tau} \int_{\nu} \phi^{2}(\tau, \nu)\left|S_{\boldsymbol{H}_{x}}(\tau, \nu)\right|^{2} d \tau d \nu}{\int_{\tau} \int_{\nu}\left|S_{\boldsymbol{H}_{x}}(\tau, \nu)\right|^{2} d \tau d \nu}\right]^{1 / 2} \\
& =\frac{1}{\left\|S_{\boldsymbol{H}_{x}}\right\|_{2}}\left[\int_{\tau} \int_{\nu} \phi^{2}(\tau, \nu)\left|S_{\boldsymbol{H}_{x}}(\tau, \nu)\right|^{2} d \tau d \nu\right]^{1 / 2}
\end{aligned}
$$

and, as special cases, by the GSF moments $m_{\boldsymbol{H}_{x}}^{(k, l)}, M_{\boldsymbol{H}_{x}}^{(k, l)}$ obtained with the weighting functions $\phi(\tau, \nu)=|\tau|^{k}|\nu|^{l}$. Thus, $\boldsymbol{H}_{x}$ is underspread if suitable weighted GSF integrals and moments are small.

The TF correlations in $x(t)$-as characterized by the GEAF $\bar{A}_{x}^{(\alpha)}(\tau, \nu)$-are related to the TF shifts introduced by $\boldsymbol{H}_{x}$. This is described by the bound [30], [76]

$$
\left|\bar{A}_{x}(\tau, \nu)\right| \leq\left|S_{\boldsymbol{H}_{x}}(\tau, \nu)\right| * *\left|S_{\boldsymbol{H}_{x}}(-\tau,-\nu)\right|
$$

${ }^{7}$ Since $S_{\boldsymbol{H}}^{\left(\alpha_{2}\right)}(\tau, \nu)=S_{\boldsymbol{H}}^{\left(\alpha_{1}\right)}(\tau, \nu) e^{j 2 \pi\left(\alpha_{1}-\alpha_{2}\right) \tau \nu}$, we henceforth use the simplified notation $\left|S_{\boldsymbol{H}}(\tau, \nu)\right| \triangleq\left|S_{\boldsymbol{H}}^{(\alpha)}(\tau, \nu)\right|$. where ** denotes two-dimensional (2-D) convolution. This bound shows that a concentrated $\left|S_{\boldsymbol{H}_{x}}(\tau, \nu)\right|$ entails a concentrated $\left|\bar{A}_{x}(\tau, \nu)\right|$, and thus an underspread innovations system $\boldsymbol{H}_{x}$ generates an underspread process $x(t)$. Conversely, if $x(t)$ is an underspread process, one can always find an underspread innovations system $\boldsymbol{H}_{x}$ [22], [102], [103]. On the other hand, the innovations system for an overspread process is necessarily overspread. This discussion shows that the weighted integrals $m_{\boldsymbol{H}_{x}}^{(\phi)}, M_{\boldsymbol{H}_{x}}^{(\phi)}$ and moments $m_{\boldsymbol{H}_{x}}^{(k, l)}, M_{\boldsymbol{H}_{x}}^{(k, l)}$ provide alternative characterizations of the "TF correlation width" and the underspread property of a process $x(t)$.

3) Simulation Examples: To illustrate these concepts, Fig. 2 shows the GEAF magnitude of underspread and overspread processes and the GSF magnitude of the associated positive semidefinite innovations system $\boldsymbol{H}_{x}=\boldsymbol{R}_{x}^{1 / 2}$. For the underspread process, the GEAF and GSF are highly concentrated about the origin. For the overspread process, however, they exhibit strong off-origin components that indicate large high-lag TF correlations. As expected from (21), the GSF of $\boldsymbol{H}_{x}$ is slightly more concentrated than the GEAF of $x(t)$.

Fig. 3 shows various type I and type II spectra for the underspread process whose GEAF is shown in Fig. 2(a). The autocorrelation function of this process was synthesized using the TF technique described in [104]. For the type II spectra, the positive semidefinite innovations system $\boldsymbol{H}_{x}=\boldsymbol{R}_{x}^{1 / 2}$ was chosen. Similarly, Fig. 4 shows the same type I and type II spectra for the overspread process of Fig. 2(c). This process was constructed by introducing strong correlations between the ' $T$ ' and ' $F$ ' components of the previous process. We will discuss and interpret these figures in the course of our subsequent development.

\section{PROPERTIES OF TyPE I SPECTRA}

By construction, all type I spectra $P_{x}^{(\mathrm{I})}(t, f)$ satisfy the superposition property (8), the TF shift covariance property (9), and the TF energy distribution property (10). Other desirable properties are satisfied if the prototype operator $\boldsymbol{P}$ underlying $P_{x}^{(\mathrm{I})}(t, f)$ satisfies certain corresponding constraints (cf. the properties and "kernel constraints" of TF representations of deterministic signals [24], [90], [91]). We now show that for an underspread process, several of these desirable properties are approximately satisfied by most type I spectra even if the corresponding constraints are not satisfied. We provide upper bounds on the associated approximation errors that allow a quantitative assessment of the accuracy of approximation. These upper bounds involve specific weighted integrals and moments of the GEAF and indicate that the approximations are accurate in the underspread case. In practice, these weighted GEAF integrals and moments can usually be reliably estimated. Proofs of the bounds presented can be found in [30]; we also note that some of the bounds are analogous to bounds presented in [27], [83] for processes with compactly supported GEAF.

\section{A. Smoothness and Statistical Cross Terms}

We first consider the GWVS $\bar{W}_{x}^{(\alpha)}(t, f)$, which is a central family of type I spectra. Since the GWVS is the 2-D Fourier transform of the GEAF (see (16)), its degree of smoothness is determined by the concentration of the GEAF about the origin. 


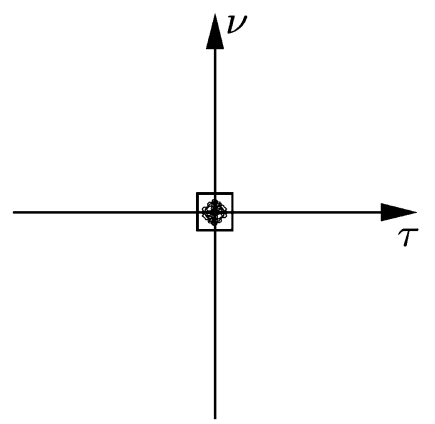

(a)

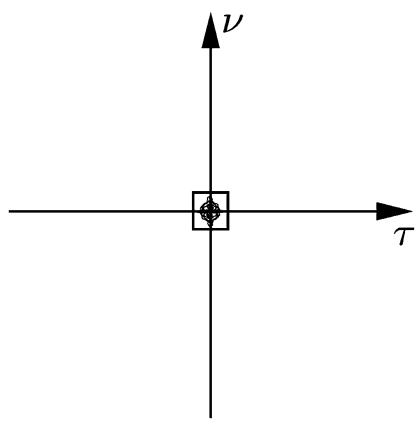

(b)

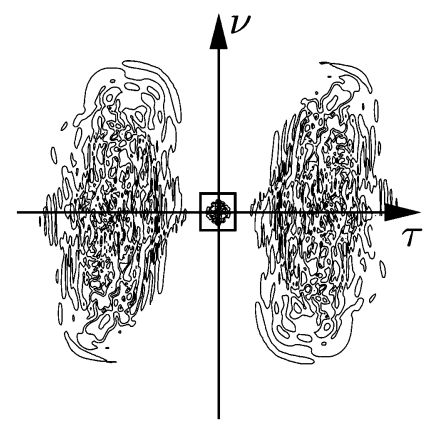

(c)

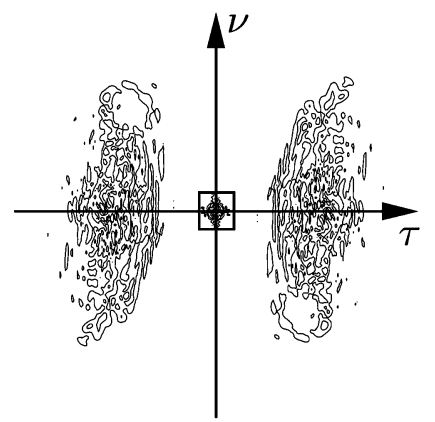

(d)

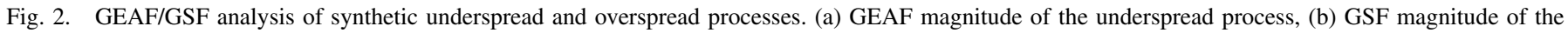

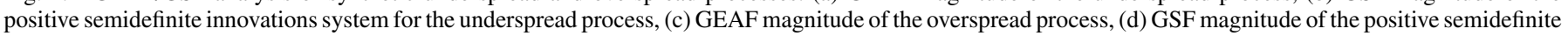

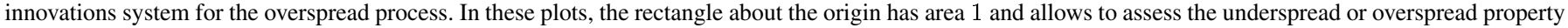
of the process under analysis.
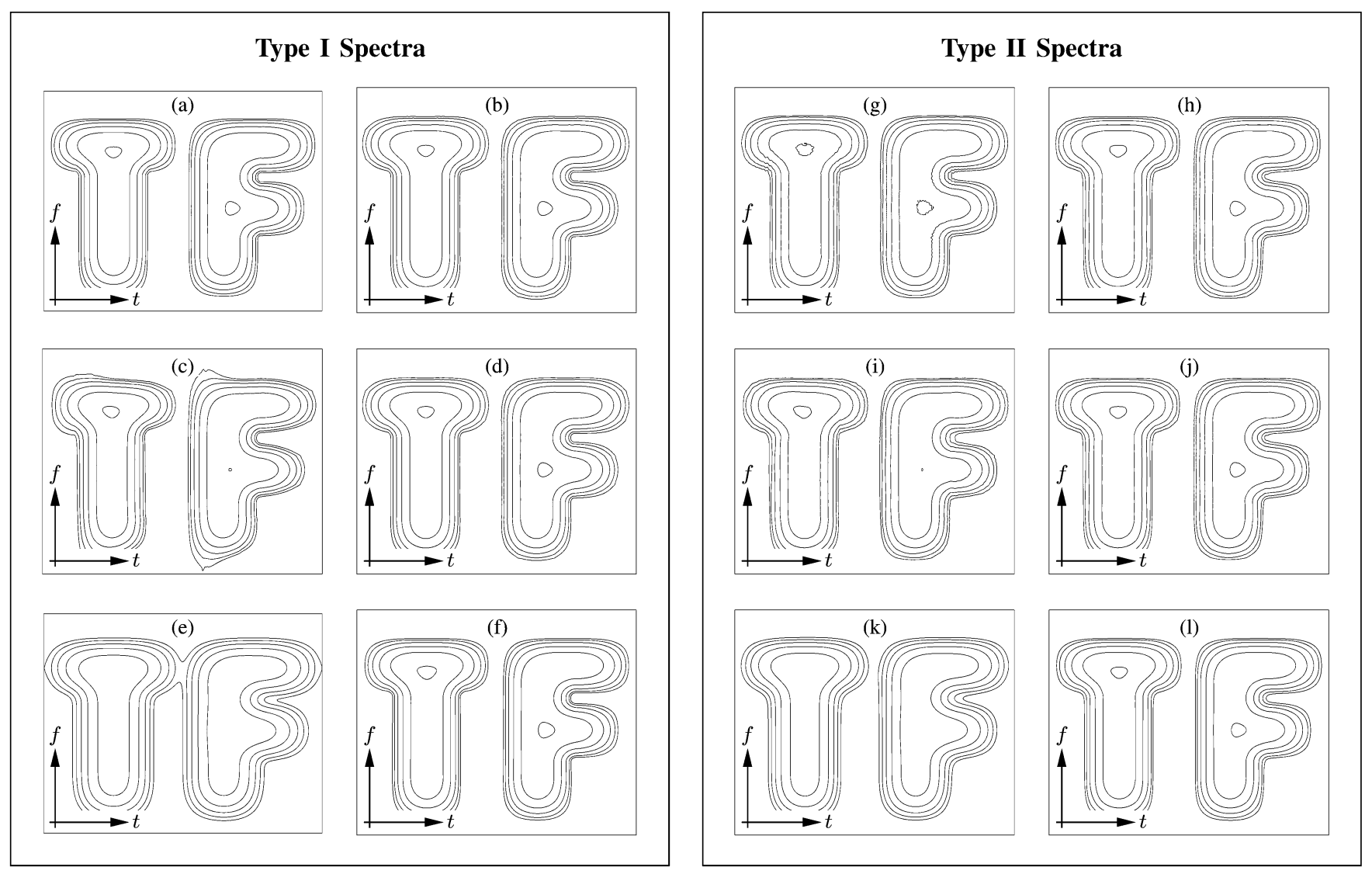

Fig. 3. Contour line plots of various type I and type II TF spectra for a synthetic underspread process. (a) Wigner-Ville spectrum (GWVS with $\alpha=0$ ), (b) real part of Rihaczek spectrum (GWVS with $\alpha=1 / 2$ ), (c) Page spectrum, (d) Levin spectrum (equal to the real part of the Rihaczek spectrum shown in (b)), (e) physical spectrum with Gaussian window, (f) multiwindow physical spectrum, (g) Weyl spectrum (GES with $\alpha=0$ ), (h) evolutionary spectrum (GES with $\alpha=1 / 2$ ), (i) type II Page spectrum, (j) type II Levin spectrum, (k) type II physical spectrum with Gaussian window, (l) type II multiwindow spectrum. The innovations system $H_{x}$ underlying the type II spectra is the positive semidefinite square root of $\boldsymbol{R}_{x}$. The multiwindow spectra use the first $N=8$ Hermite functions [24], [45] as windows and coefficients $\gamma_{1}, \ldots, \gamma_{8}$ chosen as $2.02,-1.97,1.89,-1.72,1.37,-0.89,0.42$, and -0.12 , respectively.

In particular, the GWVS of an underspread process will be a smooth 2-D lowpass function. This is demonstrated by Fig. 3(a), (b), which shows the GWVS with $\alpha=0$ and $\alpha=1 / 2$ for the underspread process whose GEAF was shown in Fig. 2(a). On the other hand, the GWVS of an overspread process will contain oscillating components. These can be seen in Fig. 4(a), (b), which shows the GWVS with $\alpha=0$ and $\alpha=1 / 2$ for the overspread process whose GEAF was shown in Fig. 2(c).
Other type I spectra $P_{x}^{(\mathrm{I})}(t, f)$ are potentially smoother than the GWVS since they are obtained from the GWVS via a convolution with $L_{\boldsymbol{P}}^{(\alpha) *}(-t,-f)$ (cf. (12a)). This convolution amounts to a smoothing if $\boldsymbol{P}$ is an underspread operator, since then $S_{\boldsymbol{P}}^{(\alpha)}(\tau, \nu)$ is concentrated about the origin and consequently $L_{\boldsymbol{P}}^{(\alpha)}(t, f)$ is a 2-D lowpass (smoothing) function. These observations are made more precise by the following result. 


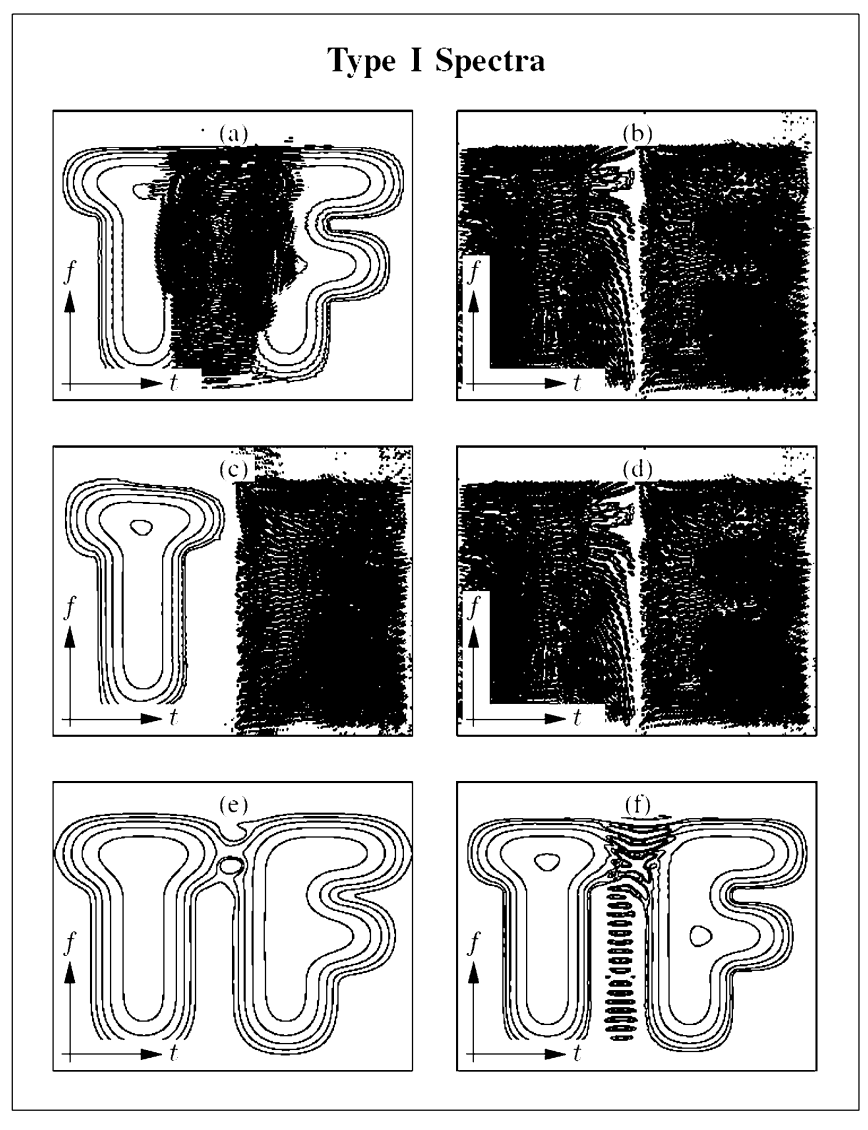

Fig. 4. Same TF spectra as in Fig. 3, but for a synthetic overspread process.

Theorem 5: The partial derivatives of any type I spectrum $P_{x}^{(\mathrm{I})}(t, f)=\left\langle\boldsymbol{R}_{x}, \boldsymbol{P}_{t, f}\right\rangle$ are bounded as

$$
\begin{gathered}
\left|\frac{\partial^{k+l} P_{x}^{(\mathrm{I})}(t, f)}{\partial t^{l} \partial f^{k}}\right| \leq(2 \pi)^{k+l} \min \left\{\delta_{1}, \delta_{2}, \delta_{3}\right\} \\
\left\|\frac{\partial^{k+l} P_{x}^{(\mathrm{I})}}{\partial t^{l} \partial f^{k}}\right\|_{2} \leq(2 \pi)^{k+l} \min \left\{\delta_{1}^{\prime}, \delta_{2}^{\prime}\right\}
\end{gathered}
$$

with

$$
\begin{aligned}
\delta_{1} & =\left\|\bar{A}_{x}\right\|_{1}\left\|S_{\boldsymbol{P}}\right\|_{\infty} m_{x}^{(k, l)} \\
\delta_{2} & =\bar{E}_{x}\left\|S_{\boldsymbol{P}}\right\|_{1} m_{\boldsymbol{P}}^{(k, l)} \\
\delta_{3} & =\left\|\bar{A}_{x}\right\|_{2}^{2}\left\|S_{\boldsymbol{P}}\right\|_{2}^{2} M_{x}^{(k p, l q)} M_{\boldsymbol{P}}^{(k(1-p), l(1-q))}
\end{aligned}
$$

(and $i$ th arbitrary $p, q \in[0,1]$ )

$$
\begin{aligned}
\delta_{1}^{\prime} & =\left\|\bar{A}_{x}\right\|_{2}\left\|S_{\boldsymbol{P}}\right\|_{\infty} M_{x}^{(k, l)} \\
\delta_{2}^{\prime} & =\bar{E}_{x}\left\|S_{\boldsymbol{P}}\right\|_{2} M_{\boldsymbol{P}}^{(k, l)} .
\end{aligned}
$$

We can draw the following conclusions.

1) If the process $x(t)$ is underspread with small moments $m_{x}^{(k, l)}$ or $M_{x}^{(k, l)}, P_{x}^{(\mathrm{I})}(t, f)$ will be smooth irrespective of the prototype operator $\boldsymbol{P}$ (this is illustrated by the examples in Fig. 3(a)-(f)).

2) If the prototype operator $\boldsymbol{P}$ is underspread with small moments $m_{\boldsymbol{P}}^{(k, l)}$ or $M_{\boldsymbol{P}}^{(k, l)}, P_{x}^{(\mathrm{I})}(t, f)$ will be smooth even if $x(t)$ is overspread (an example of such a "smoothed type I spectrum" is the physical spectrum in Fig. 4(e)).

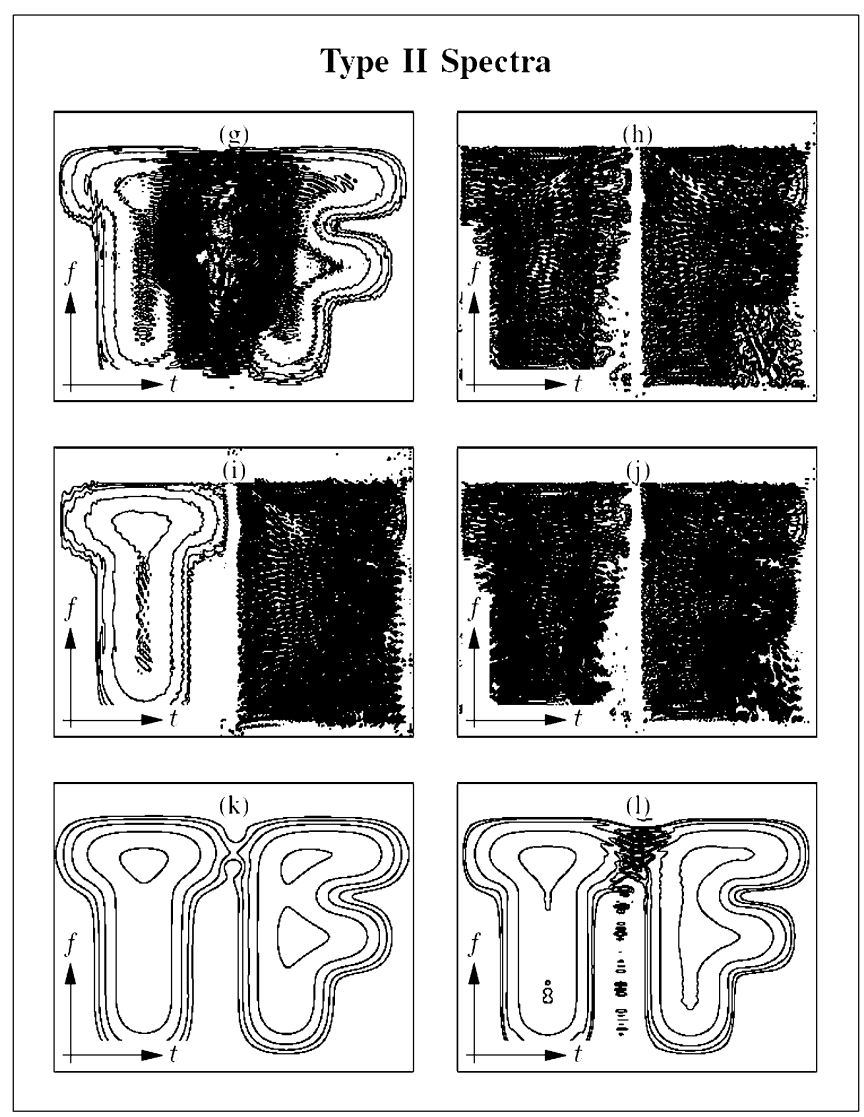

3) If neither $x(t)$ nor $\boldsymbol{P}$ is underspread, $P_{x}^{(\mathrm{I})}(t, f)$ must be expected to be nonsmooth (e.g., Fig. 4(a)-(d)).

In the last case, $P_{x}^{(\mathrm{I})}(t, f)$ typically contains strong oscillating components. These are sometimes termed statistical cross terms [29], [33]. They correspond to GEAF contributions far away from the origin, and thus indicate the presence of strong high-lag TF correlations in $x(t)$, which means that $x(t)$ is overspread. Statistical cross terms are particularly pronounced in type I spectra with $\left|S_{\boldsymbol{P}}(\tau, \nu)\right| \equiv 1$ like the GWVS and Page's spectrum, see Fig. 4(a)-(d). In contrast, for "smoothed type I spectra" (generated by underspread prototype operators $\boldsymbol{P}$ ), statistical cross terms are attenuated; this is Case 2 above.

\section{B. Real-Valuedness}

Whereas the PSD $P_{x}(f)$ is real-valued, this need not be true for a type I spectrum. According to (11), $\Im\left\{P_{x}^{(\mathrm{I})}(t, f)\right\}=0$ if and only if (iff) the operator $\boldsymbol{P}$ is self-adjoint. It can be shown that $\boldsymbol{P}$ is self-adjoint iff $S_{\boldsymbol{P}}^{(0)}(\tau, \nu)=S_{\boldsymbol{P}}^{(0) *}(-\tau,-\nu)$. The next result shows that for an underspread process $x(t), P_{x}^{(\mathrm{I})}(t, f)$ is approximately real-valued even if $\boldsymbol{P}$ is not self-adjoint.

Theorem 6: The imaginary part of any type I spectrum $P_{x}^{(\mathrm{I})}(t, f)=\left\langle\boldsymbol{R}_{x}, \boldsymbol{P}_{t, f}\right\rangle$ satisfies

$$
\frac{\left|\Im\left\{P_{x}^{(\mathrm{I})}(t, f)\right\}\right|}{\left\|\bar{A}_{x}\right\|_{1}} \leq \frac{1}{2} m_{x}^{(\phi)}, \quad \frac{\left\|\Im\left\{P_{x}^{(\mathrm{I})}\right\}\right\|_{2}}{\left\|\bar{A}_{x}\right\|_{2}}=\frac{1}{2} M_{x}^{(\phi)}
$$


with the weighting function

$$
\phi(\tau, \nu)=\left|S_{\boldsymbol{P}}^{(0)}(\tau, \nu)-S_{\boldsymbol{P}}^{(0) *}(-\tau,-\nu)\right| .
$$

This theorem shows that for underspread processes with small $m_{x}^{(\phi)}$ and $M_{x}^{(\phi)}$, we have $\Im\left\{P_{x}^{(\mathrm{I})}(t, f)\right\} \approx 0$. Due to (19), small $m_{x}^{(\phi)}$ and $M_{x}^{(\phi)}$ requires that $\phi(\tau, \nu) \approx 0$ on the effective support region of $\left|\bar{A}_{x}(\tau, \nu)\right|$, which means that

$$
S_{\boldsymbol{P}}^{(0)}(\tau, \nu) \approx S_{\boldsymbol{P}}^{(0) *}(-\tau,-\nu)
$$

on the effective support of $\left|\bar{A}_{x}(\tau, \nu)\right|$ (however, $S_{\boldsymbol{P}}^{(0)}(\tau, \nu)$ can be arbitrary outside the effective support of $\left.\left|\bar{A}_{x}(\tau, \nu)\right|\right)$. Typically, this is possible only if $\left|\bar{A}_{x}(\tau, \nu)\right|$ is sufficiently concentrated about the origin, i.e., if $x(t)$ is underspread.

Let us consider the special case of the GWVS. For the prototype operator of the GWVS, $S_{\boldsymbol{P}}^{(0)}(\tau, \nu)=e^{-j 2 \pi \alpha \tau \nu}$ and thus,

$$
\phi(\tau, \nu)=\left|e^{j 2 \pi \alpha \tau \nu}-e^{-j 2 \pi \alpha \tau \nu}\right|=2|\sin (2 \pi \alpha \tau \nu)| .
$$

Assuming $\alpha \neq 0$, it is seen that $\phi(\tau, \nu)=1$ for

$$
|\tau \nu|=(2 k+1) /(4 \alpha), \quad k \in \mathbb{N} .
$$

Thus, the condition $S_{\boldsymbol{P}}^{(0)}(\tau, \nu) \approx S_{\boldsymbol{P}}^{(0) *}(-\tau,-\nu)$ is strongly violated for certain $(\tau, \nu)$ off the origin. This shows that for overspread processes the imaginary part of the GWVS with $\alpha \neq 0$ will be significant. We furthermore note that $\phi(\tau, \nu) \leq 4 \pi|\alpha\|\tau\| \nu|$ and hence $m_{x}^{(\phi)} \leq 4 \pi|\alpha| m_{x}^{(1,1)}$ and $M_{x}^{(\phi)} \leq 4 \pi|\alpha| M_{x}^{(1,1)}$. Consequently, (22) can be replaced by the simpler but weaker bounds

$$
\begin{gathered}
\frac{\left|\Im\left\{\bar{W}_{x}^{(\alpha)}(t, f)\right\}\right|}{\left\|\bar{A}_{x}\right\|_{1}} \leq 2 \pi|\alpha| m_{x}^{(1,1)} \\
\frac{\left\|\Im\left\{\bar{W}_{x}^{(\alpha)}\right\}\right\|_{2}}{\left\|\bar{A}_{x}\right\|_{2}} \leq 2 \pi|\alpha| M_{x}^{(1,1)}
\end{gathered}
$$

which show that the GWVS will be approximately real-valued for an underspread process with small GEAF moments $m_{x}^{(1,1)}$ and $M_{x}^{(1,1)}$. Note that for $\alpha=0$, the preceding bounds are zero, which is consistent with the real-valuedness of $\bar{W}_{x}^{(0)}(t, f)$. Similar specializations and simplifications are also possible for the other results presented later.

\section{Marginal Properties}

A type I spectrum $P_{x}^{(\mathrm{I})}(t, f)$ satisfies the marginal property in time

$$
\int_{f} P_{x}^{(\mathrm{I})}(t, f) d f=\mathrm{E}\left\{|x(t)|^{2}\right\}=r_{x}(t, t)
$$

iff $S_{\boldsymbol{P}}^{(0)}(0, \nu)=1$. Similarly, the marginal property in frequency

$$
\int_{t} P_{x}^{(\mathrm{I})}(t, f) d t=\mathrm{E}\left\{|X(f)|^{2}\right\}=r_{X}(f, f)
$$

is satisfied iff $S_{\boldsymbol{P}}^{(0)}(\tau, 0)=1$. The following result shows that for an underspread process, the marginal properties are approximately satisfied even if these conditions are not met.
Theorem 7: For any type I spectrum $P_{x}^{(\mathrm{I})}(t, f)=\left\langle\boldsymbol{R}_{x}, \boldsymbol{P}_{t, f}\right\rangle$, the difference $\Delta_{1}(t) \triangleq \int_{f} P_{x}^{(\mathrm{I})}(t, f) d f-r_{x}(t, t)$ satisfies

$$
\begin{aligned}
& \frac{\left|\Delta_{1}(t)\right|}{\left\|\bar{A}_{x}\right\|_{1, \nu}} \\
& \quad \leq \tilde{m}_{x}^{(1)} \triangleq \frac{1}{\left\|\bar{A}_{x}\right\|_{1, \nu}} \int_{\nu}\left|1-S_{\boldsymbol{P}}^{(0)}(0, \nu)\right|\left|\bar{A}_{x}(0, \nu)\right| d \nu \\
& \frac{\left\|\Delta_{1}\right\|_{2}}{\left\|\bar{A}_{x}\right\|_{2, \nu}} \\
& \quad=\tilde{M}_{x}^{(1)} \triangleq \frac{1}{\left\|\bar{A}_{x}\right\|_{2, \nu}} \sqrt{\int_{\nu}\left|1-S_{\boldsymbol{P}}^{(0)}(0, \nu)\right|^{2}\left|\bar{A}_{x}(0, \nu)\right|^{2} d \nu}
\end{aligned}
$$

with $\left\|\bar{A}_{x}\right\|_{p, \nu} \triangleq\left[\int_{\nu}\left|\bar{A}_{x}(0, \nu)\right|^{p} d \nu\right]^{1 / p}, p=1,2$. Similarly, the difference $\Delta_{2}(f) \triangleq \int_{t} P_{x}^{(\mathrm{I})}(t, f) d t-r_{X}(f, f)$ satisfies

$$
\begin{aligned}
& \frac{\left|\Delta_{2}(f)\right|}{\left\|\bar{A}_{x}\right\|_{1, \tau}} \leq \\
& \tilde{m}_{x}^{(2)} \triangleq \frac{1}{\left\|\bar{A}_{x}\right\|_{1, \tau}} \int_{\tau}\left|1-S_{\boldsymbol{P}}^{(0)}(\tau, 0)\right|\left|\bar{A}_{x}(\tau, 0)\right| d \tau \\
& \frac{\left\|\Delta_{2}\right\|_{2}}{\left\|\bar{A}_{x}\right\|_{2, \tau}}= \\
& \tilde{M}_{x}^{(2)} \triangleq \frac{1}{\left\|\bar{A}_{x}\right\|_{2, \tau}} \sqrt{\int_{\tau}\left|1-S_{\boldsymbol{P}}^{(0)}(\tau, 0)\right|^{2}\left|\bar{A}_{x}(\tau, 0)\right|^{2} d \tau}
\end{aligned}
$$

with $\left\|\bar{A}_{x}\right\|_{p, \tau} \triangleq\left[\int_{\tau}\left|\bar{A}_{x}(\tau, 0)\right|^{p} d \tau\right]^{1 / p}, p=1,2$.

The weighted integrals $\tilde{m}_{x}^{(1)}$ and $\tilde{M}_{x}^{(1)}$ are measures of the extension of $\left|\bar{A}_{x}(0, \nu)\right|$, and the weighted integrals $\tilde{m}_{x}^{(2)}$ and $\tilde{M}_{x}^{(2)}$ are measures of the extension of $\left|\bar{A}_{x}(\tau, 0)\right|$. Therefore, for an underspread process where $\tilde{m}_{x}^{(1)}, \tilde{m}_{x}^{(2)}, \tilde{M}_{x}^{(1)}$ and $\tilde{M}_{x}^{(2)}$ are small (since $S_{\boldsymbol{P}}^{(0)}(0, \nu) \approx S_{\boldsymbol{P}}^{(0)}(0,0)=1$ and $S_{\boldsymbol{P}}^{(0)}(\tau, 0) \approx$ $S_{\boldsymbol{P}}^{(0)}(0,0)=1$ within the effective GEAF support), it follows from Theorem 7 that

$$
\begin{aligned}
\int_{f} P_{x}^{(\mathrm{I})}(t, f) d f & \approx \mathrm{E}\left\{|x(t)|^{2}\right\} \\
\int_{t} P_{x}^{(\mathrm{I})}(t, f) d t & \approx \mathrm{E}\left\{|X(f)|^{2}\right\} .
\end{aligned}
$$

Because $\bar{A}_{x}^{(\alpha)}(0, \nu) \leftrightarrow r_{x}(t, t)$ and $S_{\boldsymbol{P}}^{(\alpha)}(0, \nu) \leftrightarrow p(t, t)$ are Fourier transform pairs, the extension of $\left|\bar{A}_{x}(0, \nu)\right|$ determines the rate of variation of $r_{x}(t, t)$, and the condition that $S_{\boldsymbol{P}}^{(0)}(0, \nu) \approx 1$ within the GEAF support determines the extension of $p(t, t)$. Hence, small $\tilde{m}_{x}^{(1)}$ and $\tilde{M}_{x}^{(1)}$ means that the mean instantaneous power $\mathrm{E}\left\{|x(t)|^{2}\right\}=r_{x}(t, t)$ varies slowly in the sense that it is approximately constant over time spans on the order of the effective temporal width of $p(t, t)$. This interpretation can also be inferred from the relation

$$
\int_{f} P_{x}^{(\mathrm{I})}(t, f) d f=\int_{t^{\prime}} r_{x}\left(t^{\prime}, t^{\prime}\right) p^{*}\left(t-t^{\prime}, t-t^{\prime}\right) d t^{\prime}
$$

Similarly, the second approximation in (23) will hold if the mean energy density $\mathrm{E}\left\{|X(f)|^{2}\right\}=r_{X}(f, f)$ is approximately con- 
stant over frequency bands whose width is equal to the effective bandwidth of $\boldsymbol{P}$.

\section{Moyal-Type Relation}

Similarly to the Moyal property for deterministic signals (e.g., [24], [91], [105]), the Moyal-type property

$$
\left\langle P_{x}^{(\mathrm{I})}, P_{y}^{(\mathrm{I})}\right\rangle=\left\langle\boldsymbol{R}_{x}, \boldsymbol{R}_{y}\right\rangle
$$

expresses a "preservation of inner products" between the signal (correlation) domain and the TF spectrum domain. It can be shown that this property is satisfied by a type I spectrum with prototype operator $\boldsymbol{P}$ iff $\left|S_{\boldsymbol{P}}(\tau, \nu)\right| \equiv 1$. More generally, we have the following result.

Theorem 8: For any type I spectrum $P^{(\mathrm{I})}(t, f)=\left\langle\boldsymbol{R} . \boldsymbol{P}_{t, f}\right\rangle$, the difference between $\left\langle P_{x}^{(\mathrm{I})}, P_{y}^{(\mathrm{I})}\right\rangle$ and $\left\langle\boldsymbol{R}_{x}, \boldsymbol{R}_{y}\right\rangle$ is bounded as

$$
\frac{\left|\left\langle P_{x}^{(\mathrm{I})}, P_{y}^{(\mathrm{I})}\right\rangle-\left\langle\boldsymbol{R}_{x}, \boldsymbol{R}_{y}\right\rangle\right|}{\left\|\boldsymbol{R}_{x}\right\|\left\|\boldsymbol{R}_{y}\right\|} \leq \min \left\{M_{x}^{(\phi)}, M_{y}^{(\phi)}\right\}
$$

with $\phi(\tau, \nu)=\left.|1-| S_{\boldsymbol{P}}(\tau, \nu)\right|^{2} \mid$.

This theorem shows that for processes $x(t)$ and $y(t)$ where $M_{x}^{(\phi)}$ or $M_{y}^{(\phi)}$ is small, type I spectra will satisfy (24) at least approximately, i.e.,

$$
\left\langle P_{x}^{(\mathrm{I})}, P_{y}^{(\mathrm{I})}\right\rangle \approx\left\langle\boldsymbol{R}_{x}, \boldsymbol{R}_{y}\right\rangle .
$$

Small $M_{x}^{(\phi)}$ or $M_{y}^{(\phi)}$ requires that $\left|S_{\boldsymbol{P}}(\tau, \nu)\right| \approx 1$ on the effective support of $\left|\bar{A}_{x}(\tau, \nu)\right|$ or $\left|\bar{A}_{y}(\tau, \nu)\right|$ (outside the effective GEAF supports, however, $\left|S_{\boldsymbol{P}}(\tau, \nu)\right|$ can be arbitrary). This condition requires that either $x(t)$ or $y(t)$ is underspread. It is more easily satisfied for smaller effective GEAF supports, i.e., for processes that are more underspread.

For the special case $y(t)=x(t)$, we obtain

$$
\frac{\|\| P_{x}^{(\mathrm{I})}\left\|_{2}^{2}-\right\| \boldsymbol{R}_{x} \|^{2} \mid}{\left\|\boldsymbol{R}_{x}\right\|^{2}} \leq M_{x}^{(\phi)}
$$

with the weaker bound following from the Schwarz inequality. Hence, Theorem 8 also shows that for an underspread process $x(t)$ with small $M_{x}^{(\phi)}$, the $L_{2}$ norm of $P_{x}^{(\mathrm{I})}(t, f)$ approximately equals the Hilbert-Schmidt norm of the correlation operator $\boldsymbol{R}_{x}$, i.e., $\left\|P_{x}^{(\mathrm{I})}\right\|_{2} \approx\left\|\boldsymbol{R}_{x}\right\|$.

\section{E. Positivity}

The positivity (more precisely, nonnegativity) of the PSD, $P_{x}(f) \geq 0$, is crucial for the PSD's interpretation as a spectral power density. For TF spectra, too, positivity is usually considered a desirable property (however, a geometric interpretation of the Rihaczek spectrum that does not require positivity is described in [58], [99]). Wigner's theorem [24], [106] shows the nonexistence of type I spectra that are always positive and simultaneously satisfy the marginal properties. Positivity of type I spectra is also incompatible with several other desirable properties [24]. However, we will show below that type I spectra are approximately positive and may still satisfy other desirable properties for the practically relevant subclass of underspread processes.
A type I spectrum $P_{x}^{(\mathrm{I})}(t, f)$ is positive for all processes $x(t)$ iff the underlying prototype operator $\boldsymbol{P}$ is positive (semi)definite, i.e., iff $P \geq \mathbf{0}$. This is the case, e.g., for the physical spectrum and for multiwindow physical spectra with $\gamma_{k} \geq 0$, but not for the Wigner-Ville spectrum $\bar{W}_{x}^{(0)}(t, f)$. However, in [16], [23], [98] it was conjectured and demonstrated by means of examples that the Wigner-Ville spectrum of a "sufficiently random" process is effectively positive. Interpreting the notion of "sufficient randomness" as a lack of strong TF correlations, we immediately obtain a link to our underspread concept.

We now demonstrate that the type I spectra of underspread processes indeed are effectively positive, in addition to being effectively real-valued as shown in Section IV-B. We can split $P_{x}^{(\mathrm{I})}(t, f)$ as

$P_{x}^{(\mathrm{I})}(t, f)=\mathcal{P}\left\{P_{x}^{(\mathrm{I})}(t, f)\right\}+\mathcal{N}\left\{P_{x}^{(\mathrm{I})}(t, f)\right\}+j \Im\left\{P_{x}^{(\mathrm{I})}(t, f)\right\}$

where $\mathcal{P}\left\{P_{x}^{(\mathrm{I})}(t, f)\right\}$ and $\mathcal{N}\left\{P_{x}^{(\mathrm{I})}(t, f)\right\}$ are, respectively, the positive real part and negative real part of $P_{x}^{(\mathrm{I})}(t, f)$ which are defined by

$$
\begin{aligned}
\mathcal{P}\left\{P_{x}^{(\mathrm{I})}(t, f)\right\} & \triangleq \frac{\Re\left\{P_{x}^{(\mathrm{I})}(t, f)\right\}+\left|\Re\left\{P_{x}^{(\mathrm{I})}(t, f)\right\}\right|}{2} \\
\mathcal{N}\left\{P_{x}^{(\mathrm{I})}(t, f)\right\} & \triangleq \frac{\Re\left\{P_{x}^{(\mathrm{I})}(t, f)\right\}-\left|\Re\left\{P_{x}^{(\mathrm{I})}(t, f)\right\}\right|}{2} .
\end{aligned}
$$

Positivity of $P_{x}^{(\mathrm{I})}(t, f)$ means $P_{x}^{(\mathrm{I})}(t, f)=\mathcal{P}\left\{P_{x}^{(\mathrm{I})}(t, f)\right\}$ or equivalently, $\mathcal{N}\left\{P_{x}^{(\mathrm{I})}(t, f)\right\}=\Im\left\{P_{x}^{(\mathrm{I})}(t, f)\right\}=0$. Theorem 6 stated that for an underspread process $P_{x}^{(\mathrm{I})}(t, f)$ is approximately real-valued, i.e., $\Im\left\{P_{x}^{(\mathrm{I})}(t, f)\right\} \approx 0$. We now complement that result by establishing an upper bound on the negative real part $\mathcal{N}\left\{P_{x}^{(\mathrm{I})}(t, f)\right\}$.

Theorem 9: The negative real part $\mathcal{N}\left\{P_{x}^{(\mathrm{I})}(t, f)\right\}$ of any type I spectrum $P_{x}^{(\mathrm{I})}(t, f)=\left\langle\boldsymbol{R}_{x}, \boldsymbol{P}_{t, f}\right\rangle$ is bounded as

$$
\begin{aligned}
\frac{\left|\mathcal{N}\left\{P_{x}^{(\mathrm{I})}(t, f)\right\}\right|}{\left\|\bar{A}_{x}\right\|_{1}} & \leq \inf _{\boldsymbol{P}^{\prime} \geq \mathbf{0}}\left\{m_{x}^{\left(\phi_{\boldsymbol{P}^{\prime}}\right)}\right\} \\
\frac{\left\|\mathcal{N}\left\{P_{x}^{(\mathrm{I})}\right\}\right\|_{2}}{\left\|\bar{A}_{x}\right\|_{2}} & \leq \inf _{\boldsymbol{P}^{\prime} \geq \mathbf{0}}\left\{M_{x}^{\left(\phi_{\boldsymbol{P}^{\prime}}\right)}\right\}
\end{aligned}
$$

with $\phi_{\boldsymbol{P}^{\prime}}(\tau, \nu)=\left|S_{\boldsymbol{P}}^{(\alpha)}(\tau, \nu)-S_{\boldsymbol{P}^{\prime}}^{(\alpha)}(\tau, \nu)\right|$.

This theorem shows that for an underspread process where $m_{x}^{\left(\phi_{\boldsymbol{P}^{\prime}}\right)}$ and $M_{x}^{\left(\phi_{\boldsymbol{P}^{\prime}}\right)}$ are small for some positive (semi)definite $\boldsymbol{P}^{\prime}$, the negative real part of $P_{x}^{(\mathrm{I})}(t, f)$ is approximately zero, i.e., $\mathcal{N}\left\{P_{x}^{(\mathrm{I})}(t, f)\right\} \approx 0$. If $P_{x}^{(\mathrm{I})}(t, f)$ is indeed positive $(\boldsymbol{P} \geq \mathbf{0})$, the bounds (25) will be zero since with $\boldsymbol{P}^{\prime}=\boldsymbol{P}$ we have $m_{x}^{\left(\phi_{\boldsymbol{P}^{\prime}}\right)}=M_{x}^{\left(\phi_{\boldsymbol{P}^{\prime}}\right)}=0$. Otherwise, small $\inf _{\boldsymbol{P}^{\prime} \geq \mathbf{0}} m_{x}^{\left(\phi_{\boldsymbol{P}^{\prime}}\right)}$ and $\inf _{\boldsymbol{P}^{\prime} \geq \mathbf{0}} M_{x}^{\left(\phi_{\boldsymbol{P}^{\prime}}\right)}$ requires that, on the effective support of $\bar{A}_{x}^{(\alpha)}(\tau, \nu), S_{\boldsymbol{P}}^{(\alpha)}(\tau, \nu)$ can be well approximated by the GSF $S_{\boldsymbol{P}^{\prime}}^{(\alpha)}(\tau, \nu)$ of a positive semidefinite operator $\boldsymbol{P}^{\prime}$. This is favored by underspread processes where the effective support of $\bar{A}_{x}^{(\alpha)}(\tau, \nu)$ is small.

By combining Theorems 6 and 9 by means of the triangle inequality, we obtain the following bounds on the difference between $P_{x}^{(\mathrm{I})}(t, f)$ and its positive real part $\mathcal{P}\left\{P_{x}^{(\mathrm{I})}(t, f)\right\}$. 
Corollary 10: For any type I spectrum $P_{x}^{(\mathrm{I})}(t, f)=$ $\left\langle\boldsymbol{R}_{x}, \boldsymbol{P}_{t, f}\right\rangle$, the difference between $P_{x}^{(\mathrm{I})}(t, f)$ and $\mathcal{P}\left\{P_{x}^{(\mathrm{I})}(t, f)\right\}$ is bounded as

$$
\begin{aligned}
\frac{\left|P_{x}^{(\mathrm{I})}(t, f)-\mathcal{P}\left\{P_{x}^{(\mathrm{I})}(t, f)\right\}\right|}{\left\|\bar{A}_{x}\right\|_{1}} & \leq \frac{1}{2} m_{x}^{(\phi)}+\inf _{\boldsymbol{P}^{\prime} \geq \mathbf{0}}\left\{m_{x}^{\left(\phi_{\boldsymbol{P}^{\prime}}\right)}\right\} \\
\frac{\left\|P_{x}^{(\mathrm{I})}-\mathcal{P}\left\{P_{x}^{(\mathrm{I})}\right\}\right\|_{2}}{\left\|\bar{A}_{x}\right\|_{2}} & \leq \frac{1}{2} M_{x}^{(\phi)}+\inf _{\boldsymbol{P}^{\prime} \geq \mathbf{0}}\left\{M_{x}^{\left(\phi_{\boldsymbol{P}^{\prime}}\right)}\right\}
\end{aligned}
$$

with

and

$$
\phi(\tau, \nu)=\left|S_{\boldsymbol{P}}^{(0)}(\tau, \nu)-S_{\boldsymbol{P}}^{(0) *}(-\tau,-\nu)\right|
$$

$$
\phi_{\boldsymbol{P}^{\prime}}(\tau, \nu)=\left|S_{\boldsymbol{P}}^{(\alpha)}(\tau, \nu)-S_{\boldsymbol{P}^{\prime}}^{(\alpha)}(\tau, \nu)\right|
$$

This corollary finally shows that type I spectra of underspread processes (where $m_{x}^{(\phi)}, M_{x}^{(\phi)}, \inf _{\boldsymbol{P}^{\prime}>\mathbf{0}} m_{x}^{\left(\phi_{\boldsymbol{P}^{\prime}}\right)}$, and $\inf _{P^{\prime} \geq \mathbf{0}} M_{x}^{\left(\phi_{P^{\prime}}\right)}$ are small) are approximately real-valued and positive, i.e., $P_{x}^{(\mathrm{I})}(t, f) \approx \mathcal{P}\left\{P_{x}^{(\mathrm{I})}(t, f)\right\}$ or equivalently $\Im\left\{P_{x}^{(\mathrm{I})}(t, f)\right\} \approx 0$ and $\mathcal{N}\left\{P_{x}^{(\mathrm{I})}(t, f)\right\} \approx 0$. These approximations require that within the effectice GEAF support, the spreading function of the prototype operator $P$ be nearly Hermitian symmetric and close to that of a positive operator, whereas it may be arbitrary outside the effective GEAF support.

\section{Properties of Type II SPECTRA}

Next, we consider the properties of type II spectra. By construction, all type II spectra $P_{x}^{(\mathrm{II})}(t, f)$ are TF shift covariant and positive. Other desirable properties are satisfied if the prototype operator $\boldsymbol{P}$ satisfies corresponding constraints. In this section, we show that for underspread processes, some desirable properties are approximately satisfied by most type II spectra even if the corresponding constraints are not satisfied. As in Section IV, we provide upper bounds on the associated approximation errors.

\section{A. Smoothness and Statistical Cross Terms}

We first consider the GES (17), which is a central family of type II spectra. Due to $G_{x}^{(\alpha)}(t, f)=\left|L_{\boldsymbol{H}_{x}}^{(\alpha)}(t, f)\right|^{2}$, the smoothness of the GES is determined by the smoothness of the GWS $L_{\boldsymbol{H}_{x}}^{(\alpha)}(t, f)$ of the innovations system $\boldsymbol{H}_{x}$, which in turn is determined by the concentration of the GSF $S_{\boldsymbol{H}_{x}}^{(\alpha)}(\tau, \nu)$. Therefore, if the innovations system $\boldsymbol{H}_{x}$-and thus, $x(t)$ itself-is underspread, the GES will be a smooth 2-D lowpass function. Examples are shown in Fig. 3(g), (h). In contrast, if the innovations system is overspread, which typically produces an overspread process, the GES will contain oscillating components. This is shown in Fig. 4(g), (h).

Other type II spectra are potentially smoother than the GES because according to (15), any type II spectrum involves a convolution of $L_{\boldsymbol{H}_{x}}^{(\alpha)}(t, f)$ with $L_{\boldsymbol{P}}^{(\alpha) *}(-t,-f)$. This convolution amounts to a smoothing if $\boldsymbol{P}$ is an underspread operator, because then $L_{\boldsymbol{P}}^{(\alpha)}(t, f)$ is a smooth 2-D lowpass function. These observations are made more precise by the following result.
Theorem 11: The partial derivatives of any type II spectrum $P_{x}^{(\mathrm{II})}(t, f)=\left|\left\langle\boldsymbol{H}_{x}, \boldsymbol{P}_{t, f}\right\rangle\right|^{2}$ are bounded as

$$
\left|\frac{\partial^{k+l} P_{x}^{(\mathrm{II})}(t, f)}{\partial t^{l} \partial f^{k}}\right| \leq(2 \pi)^{k+l} \tilde{\delta}
$$

where

$$
\tilde{\delta}=\min \left\{\left\|S_{\boldsymbol{H}_{x}}\right\|_{1}^{2}\left\|S_{\boldsymbol{P}}\right\|_{\infty}^{2} \bar{m}_{\boldsymbol{H}_{x}}^{(k, l)},\left\|S_{\boldsymbol{H}_{x}}\right\|_{\infty}^{2}\left\|S_{\boldsymbol{P}}\right\|_{1}^{2} \bar{m}_{\boldsymbol{P}}^{(k, l)}\right\}
$$

with

$$
\bar{m}_{\boldsymbol{H}}^{(k, l)} \triangleq \sum_{i=0}^{k} \sum_{j=0}^{l}\left(\begin{array}{l}
k \\
i
\end{array}\right)\left(\begin{array}{l}
l \\
j
\end{array}\right) m_{\boldsymbol{H}}^{(i, j)} m_{\boldsymbol{H}}^{(k-i, l-j)}
$$

This shows that $P_{x}^{(\mathrm{II})}(t, f)$ is smooth either when the innovations system $\boldsymbol{H}_{x}$ has small convolved moments $\bar{m}_{\boldsymbol{H}_{x}}^{(k, l)}$, or when the prototype operator $\boldsymbol{P}$ has small convolved moments $\bar{m}_{\boldsymbol{P}}^{(k, l)}$. These convolved moments will be small when the GSF moments $m_{\boldsymbol{H}_{x}}^{(k, l)}$ or $m_{\boldsymbol{P}}^{(k, l)}$ are themselves small. We can draw the following conclusions.

1) If $\boldsymbol{H}_{x}$-and thus $x(t)$-is underspread with small moments $m_{\boldsymbol{H}_{x}}^{(k, l)}, P_{x}^{(\mathrm{II})}(t, f)$ will be smooth irrespective of the prototype operator $\boldsymbol{P}$ (this is the case e.g., in Fig. $3(\mathrm{~g})-(\mathrm{l}))$.

2) If the prototype operator $P$ is underspread with small moments $m_{\boldsymbol{P}}^{(k, l)}, P_{x}^{(\mathrm{II})}(t, f)$ will be smooth even for an overspread innovations system $\boldsymbol{H}_{x}$ (an example of such a "smoothed type II spectrum" is the type II physical spectrum shown in Fig. 4(k)).

3) If neither $\boldsymbol{H}_{x}$ nor $\boldsymbol{P}$ is underspread, $P_{x}^{(\mathrm{II})}(t, f)$ will not be smooth.

In the last case, $P_{x}^{(\mathrm{II})}(t, f)$ typically contains strong oscillating components (statistical cross terms [29]) that correspond to components of $S_{\boldsymbol{H}_{x}}^{(\alpha)}(\tau, \nu)$ far away from the origin. These components indicate large TF shifts effected by $\boldsymbol{H}_{x}$ and thus, typically, strong high-lag TF correlations in $x(t)$-i.e., $x(t)$ is overspread. The statistical cross terms of type II spectra are different from those of type I spectra in that they do not lead to negative values of $P_{x}^{(\mathrm{II})}(t, f)$. Examples are shown in Fig. $4(\mathrm{~g})-(\mathrm{j})$. However, statistical cross terms are attenuated or suppressed in "smoothed type II spectra" (cf. Case 2 above). Note that, according to (15), the smoothing is applied to the GWS $L_{\boldsymbol{H}_{x}}^{(\alpha)}(t, f)$, i.e., before taking the squared magnitude.

\section{B. Mean Energy}

It can be shown that in order for a type II spectrum to integrate to the mean energy of the process, i.e.,

$$
\int_{t} \int_{f} P_{x}^{(\mathrm{II})}(t, f) d t d f=\bar{E}_{x}
$$

the prototype operator $\boldsymbol{P}$ has to satisfy $\left|S_{\boldsymbol{P}}(\tau \nu)\right| \equiv 1$. The following result shows that for an underspread innovations system $\boldsymbol{H}_{x}$, (26) is satisfied at least approximately even if $\left|S_{\boldsymbol{P}}(\tau \nu)\right| \not \equiv 1$. 
Theorem 12: For any type II spectrum $P_{x}^{(\mathrm{II})}(t, f)=$ $\left|\left\langle\boldsymbol{H}_{x}, \boldsymbol{P}_{t, f}\right\rangle\right|^{2}$, the difference $\Delta \triangleq \int_{t} \int_{f} P_{x}^{(\mathrm{II})}(t, f) d t d f-\bar{E}_{x}$ is bounded as

$$
\frac{\Delta}{\left\|\boldsymbol{H}_{x}\right\|^{2}} \leq\left[M_{\boldsymbol{H}_{x}}^{(\phi)}\right]^{2}
$$

where $\phi(\tau, \nu)=\sqrt{\left.|1-| S_{\boldsymbol{P}}(\tau, \nu)\right|^{2} \mid}$.

Thus, for an innovations system $\boldsymbol{H}_{x}$ with small $M_{\boldsymbol{H}_{x}}^{(\phi)}$, the integral of $P_{x}^{(\mathrm{II})}(t, f)$ is approximately equal to the mean energy, i.e.,

$$
\int_{t} \int_{f} P_{x}^{(\mathrm{II})}(t, f) d t d f \approx \bar{E}_{x}
$$

The weighted GSF integral $M_{\boldsymbol{H}_{x}}^{(\phi)}$ will be small if $\left|S_{\boldsymbol{P}}(\tau, \nu)\right| \approx 1$ on the effective support of $\left|S_{\boldsymbol{H}_{x}}(\tau, \nu)\right|$. This is favored by a small effective support of $\left|S_{\boldsymbol{H}_{x}}(\tau, \nu)\right|$, i.e., by an underspread innovations system and, hence, by an underspread process $x(t)$.

\section{Marginal Properties}

A type II spectrum $P_{x}^{(\mathrm{II})}(t, f)$ satisfies the marginal property in time

$$
\int_{f} P_{x}^{(\mathrm{II})}(t, f) d f=r_{x}(t, t)
$$

iff $S_{\boldsymbol{P}}^{(1 / 2)}(\tau, \nu) S_{\boldsymbol{P}}^{(1 / 2) *}\left(\tau, \nu-\nu^{\prime}\right) \equiv 1$ or, equivalently, iff $S_{\boldsymbol{P}}^{(1 / 2)}(\tau, \nu)=e^{j \phi(\tau)}$, where $\phi(\tau)$ is an arbitrary phase function (this condition is satisfied, e.g., by the evolutionary spectrum, where $\left.S_{\boldsymbol{P}}^{(1 / 2)}(\tau, \nu) \equiv 1\right)$. Similarly, $P_{x}^{(\mathrm{II})}(t, f)$ satisfies the marginal property in frequency

$$
\int_{t} P_{x}^{(\mathrm{II})}(t, f) d t=r_{X}(f, f)
$$

iff $S_{\boldsymbol{P}}^{(-1 / 2)}(\tau, \nu) S_{\boldsymbol{P}}^{(-1 / 2) *}\left(\tau-\tau^{\prime}, \nu\right) \equiv 1$ or $S_{\boldsymbol{P}}^{(-1 / 2)}(\tau, \nu)=$ $e^{j \Psi(\nu)}$ (this is satisfied e.g., by the transitory evolutionary spectrum, where $\left.S_{P}^{(-1 / 2)}(\tau, \nu) \equiv 1\right)$. We note that the evolutionary and transitory evolutionary spectra are equal when computed using the same self-adjoint innovation system [22]. In that case, one obtains a positive spectrum satisfying both marginals. This is not a contradiction to Wigner's theorem [24], [106] since this spectrum is not the expectation of a quadratic TF signal representation.

Explicit expressions for the differences

$\int_{f} P_{x}^{(\mathrm{II})}(t, f) d f-r_{x}(t, t)$ and $\int_{t} P_{x}^{(\mathrm{II})}(t, f) d t-r_{X}(f, f)$

exist but are rather complicated. Thus, here we content ourselves with bounding these differences for the GES, i.e., for $P_{x}^{(\mathrm{II})}(t, f)=G_{x}^{(\alpha)}(t, f)=\left|L_{\boldsymbol{H}_{x}}^{(\alpha)}(t, f)\right|^{2}$ (here, $S_{\boldsymbol{P}}^{(\alpha)}(\tau, \nu) \equiv 1$ ). Our bounds extend previous results in [22].

Theorem 13: The differences

$$
\begin{aligned}
& \Delta_{1}(t) \triangleq \int_{f} G_{x}^{(\alpha)}(t, f) d f-r_{x}(t, t) \\
& \Delta_{2}(f) \triangleq \int_{t} G_{x}^{(\alpha)}(t, f) d t-r_{X}(f, f)
\end{aligned}
$$

are bounded as

$$
\begin{aligned}
& \frac{\left|\Delta_{1}(t)\right|}{\left\|S_{\boldsymbol{H}_{x}}\right\|_{\infty, 1}\left\|S_{\boldsymbol{H}_{x}}\right\|_{1}} \leq 4 \pi\left|\alpha-\frac{1}{2}\right| m_{\boldsymbol{H}_{x}}^{(1,1)} \\
& \frac{\left|\Delta_{2}(f)\right|}{\left\|S_{\boldsymbol{H}_{x}}\right\|_{1, \infty}\left\|S_{\boldsymbol{H}_{x}}\right\|_{1}} \leq 4 \pi\left|\alpha+\frac{1}{2}\right| m_{\boldsymbol{H}_{x}}^{(1,1)}
\end{aligned}
$$

with

$$
\begin{aligned}
& \left\|S_{\boldsymbol{H}_{x}}\right\|_{\infty, 1} \triangleq \sup _{\tau}\left\{\int_{\nu}\left|S_{\boldsymbol{H}_{x}}(\tau, \nu)\right| d \nu\right\} \\
& \left\|S_{\boldsymbol{H}_{x}}\right\|_{1, \infty} \triangleq \sup _{\nu}\left\{\int_{\tau}\left|S_{\boldsymbol{H}_{x}}(\tau, \nu)\right| d \tau\right\} .
\end{aligned}
$$

This theorem shows that for an underspread innovations system with small $m_{\boldsymbol{H}_{x}}^{(1,1)}$ (which implies that $x(t)$ is underspread), the GES approximately satisfies the marginal properties, i.e.,

$\int_{f} G_{x}^{(\alpha)}(t, f) d f \approx r_{x}(t, t)$ and $\int_{t} G_{x}^{(\alpha)}(t, f) d t \approx r_{X}(f, f)$.

Small $m_{\boldsymbol{H}_{x}}^{(1,1)}$ requires $\left|S_{\boldsymbol{H}_{x}}(\tau, \nu)\right|$ to be concentrated along the $\tau$ and $\nu$ axes. The bounds in (27) and (28) correctly reflect the fact that the marginal property in time is exactly satisfied by the evolutionary spectrum (GES with $\alpha=1 / 2$ ) and the marginal property in frequency is exactly satisfied by the transitory evolutionary spectrum (GES with $\alpha=-1 / 2$ ).

\section{APPRoXimate EQuivalenCE OF TF SPECTRA}

Within both the type I and type II classes, an infinite number of different TF spectra can be obtained by different choices of the prototype operator $\boldsymbol{P}$. This nonuniqueness is a significant—and often inconvenient-difference from the stationary case. One may, of course, advocate a specific TF spectrum because it satisfies a given set of properties not satisfied by any other spectrum. However, we next demonstrate that the various type I and type II spectra will give effectively equivalent results for underspread processes. Thus, in the underspread case, it does not make much difference which TF spectrum is used.

\section{A. Approximate Equivalences Within the Type I and Type II Classes}

The next theorem considers the pointwise and $L_{2}$ deviations between two different type I spectra.

Theorem 14: The difference of two type I spectra $P_{x}^{(\mathrm{I})}(t, f)=\left\langle\boldsymbol{R}_{x}, \boldsymbol{P}_{t, f}\right\rangle$ and $\tilde{P}_{x}^{(\mathrm{I})}(t, f)=\left\langle\boldsymbol{R}_{x}, \tilde{\boldsymbol{P}}_{t, f}\right\rangle$ satisfies

$$
\begin{aligned}
& \frac{\left|P_{x}^{(\mathrm{I})}(t, f)-\tilde{P}_{x}^{(\mathrm{I})}(t, f)\right|}{\left\|\bar{A}_{x}\right\|_{1}} \leq m_{x}^{(\phi)} \\
& \frac{\left\|P_{x}^{(\mathrm{I})}-\tilde{P}_{x}^{(\mathrm{I})}\right\|_{2}}{\left\|\bar{A}_{x}\right\|_{2}}=M_{x}^{(\phi)}
\end{aligned}
$$

where $\phi(\tau, \nu)=\left|S_{\boldsymbol{P}}^{(\alpha)}(\tau, \nu)-S_{\tilde{\boldsymbol{P}}}^{(\alpha)}(\tau, \nu)\right|$.

Thus, for underspread processes with small $m_{x}^{(\phi)}$ and $M_{x}^{(\phi)}$, different type I spectra $P_{x}^{(\mathrm{I})}(t, f)$ and $\tilde{P}_{x}^{(\mathrm{I})}(t, f)$ are approximately equal, i.e.,

$$
P_{x}^{(\mathrm{I})}(t, f) \approx \tilde{P}_{x}^{(\mathrm{I})}(t, f) .
$$


Small $m_{x}^{(\phi)}$ and $M_{x}^{(\phi)}$ requires that $\phi(\tau, \nu) \approx 0$ or, equivalently, $S_{\boldsymbol{P}}^{(\alpha)}(\tau, \nu) \approx S_{\tilde{\boldsymbol{P}}}^{(\alpha)}(\tau, \nu)$ on the effective support of the GEAF $\bar{A}_{x}^{(\alpha)}(\tau, \nu)$ (however, $S_{\boldsymbol{P}}^{(\alpha)}(\tau, \nu)$ may be completely different from $S_{\tilde{P}}^{(\alpha)}(\tau, \nu)$ outside the effective GEAF support). This is favored by an underspread process, where the effective GEAF support is small.

As an example, consider the GWVS and the physical spectrum, i.e.,

$$
P_{x}^{(\mathrm{I})}(t, f)=\bar{W}_{x}^{(\alpha)}(t, f) \quad \text { and } \quad \tilde{P}_{x}^{(\mathrm{I})}(t, f)=\operatorname{PS}_{x}^{(g)}(t, f) .
$$

Here, we have $S_{\boldsymbol{P}}^{(\alpha)}(\tau, \nu)=1$ and $S_{\tilde{\boldsymbol{P}}}^{(\alpha)}(\tau, \nu)=A_{g}^{(\alpha) *}(\tau, \nu)$ so that the weighting function is $\phi(\tau, \nu)=\left|1-A_{g}^{(\alpha)}(\tau, \nu)\right|$. A Taylor series expansion of $A_{g}^{(\alpha)}(\tau, \nu)$ about the origin shows that for $g(t)$ real and symmetric and for small $\tau$ and $\nu$, there is

$$
\begin{array}{r}
\left|1-A_{g}^{(\alpha)}(\tau, \nu)\right| \approx\left|1-\left[1-\left(2 \pi F_{g} \tau\right)^{2}-\left(2 \pi T_{g} \nu\right)^{2}\right]\right| \\
=4 \pi^{2}\left(F_{g}^{2} \tau^{2}+T_{g}^{2} \nu^{2}\right) \approx 0
\end{array}
$$

where $T_{g}$ and $F_{g}$ denote the root-mean square (RMS) duration and bandwidth of $g(t)$, respectively. In contrast, for large $\tau$ and $\nu$ one has

$$
\phi(\tau, \nu)=\left|1-A_{g}^{(\alpha)}(\tau, \nu)\right| \approx 1
$$

Hence, the GWVS and physical spectrum yield similar results for underspread processes even though they may be very different otherwise.

The approximate equivalence of different type I spectra in the underspread case is clearly demonstrated by the examples depicted in Fig. 3(a)-(f). In contrast, Fig. 4(a)-(f) shows that the type I spectra may be quite different in the overspread case.

A similar equivalence result holds for the class of type II spectra, as stated by the next theorem.

Theorem 15: The difference of two type II spectra $P_{x}^{(\mathrm{II})}(t, f)=\left|\left\langle\boldsymbol{H}_{x}, \boldsymbol{P}_{t, f}\right\rangle\right|^{2}$ and $\tilde{P}_{x}^{(\mathrm{II})}(t, f)=\left|\left\langle\boldsymbol{H}_{x}, \tilde{\boldsymbol{P}}_{t, f}\right\rangle\right|^{2}$ is bounded as

$$
\frac{\left|P_{x}^{(\mathrm{II})}(t, f)-\tilde{P}_{x}^{(\mathrm{II})}(t, f)\right|}{\left\|S_{\boldsymbol{H}_{x}}\right\|_{1}^{2}} \leq 2 m_{\boldsymbol{H}_{x}}^{(\phi)}
$$

where $\phi(\tau, \nu)=\left|S_{\boldsymbol{P}}^{(\alpha)}(\tau, \nu)-S_{\tilde{\boldsymbol{P}}}^{(\alpha)}(\tau, \nu)\right|$.

Thus, for an underspread innovations system $\boldsymbol{H}_{x}$ with small $m_{\boldsymbol{H}_{x}}^{(\phi)}$, different type II spectra $P_{x}^{(\mathrm{II})}(t, f)$ and $\tilde{P}_{x}^{(\mathrm{II})}(t, f)$ based on this innovations system are approximately equal, i.e.,

$$
P_{x}^{(\mathrm{II})}(t, f) \approx \tilde{P}_{x}^{(\mathrm{II})}(t, f) .
$$

A small $m_{\boldsymbol{H}_{x}}^{(\phi)}$ requires that $S_{\boldsymbol{P}}^{(\alpha)}(\tau, \nu) \approx S_{\tilde{\boldsymbol{P}}}^{(\alpha)}(\tau, \nu)$ on the effective support of $\left|S_{\boldsymbol{H}_{x}}(\tau, \nu)\right|$. This is favored when the effective support of $\left|S_{\boldsymbol{H}_{x}}(\tau, \nu)\right|$ is small, i.e., when $\boldsymbol{H}_{x}$-and, thus, $x(t)$-is underspread. The similarity of different type II spectra in the underspread case is demonstrated in Fig. 3(g)-(l), whereas their dissimilarity in the overspread case is evident from Fig. 4(g)-(1).

\section{B. Approximate Equivalence of Type I and Type II Spectra}

We have just seen that in the underspread case, different TF spectra within the type I class or within the type II class yield similar results. It remains to study if, in addition, type I spectra are similar to type II spectra. A type I spectrum is a TF symbol of the "squared" innovations system $\boldsymbol{H}_{x} \boldsymbol{H}_{x}^{+}=\boldsymbol{R}_{x}$

$$
P_{x}^{(\mathrm{I})}(t, f)=L_{\boldsymbol{H}_{x} \boldsymbol{H}_{x}^{+}}^{(\boldsymbol{P})}(t, f)=\left\langle\boldsymbol{H}_{x} \boldsymbol{H}_{x}^{+}, \boldsymbol{P}_{t, f}\right\rangle
$$

whereas a type II spectrum is the squared magnitude of a TF symbol of the innovations system $\boldsymbol{H}_{x}$

$$
P_{x}^{(\mathrm{II})}(t, f)=\left|L_{\boldsymbol{H}_{x}}^{(\boldsymbol{P})}(t, f)\right|^{2}=\left|\left\langle\boldsymbol{H}_{x}, \boldsymbol{P}_{t, f}\right\rangle\right|^{2} .
$$

Thus, type I and type II spectra (using the same prototype operator $\boldsymbol{P}$ ) are approximately equal if the TF symbol of " $\boldsymbol{H}_{x}$ squared" is approximately equal to the squared magnitude of the TF symbol of $\boldsymbol{H}_{x}$. In the underspread case, the existence of such an approximation is confirmed by the following result that even admits different prototype operators.

Theorem 16: The difference between any type I spectrum $P_{x}^{(\mathrm{I})}(t, f)=\left\langle\boldsymbol{R}_{x}, \boldsymbol{P}_{t, f}\right\rangle$ and any type II spectrum $P_{x}^{(\mathrm{II})}(t, f)=$ $\left|\left\langle\boldsymbol{H}_{x}, \tilde{\boldsymbol{P}}_{t, f}\right\rangle\right|^{2}$ is bounded as

$$
\frac{\left|P_{x}^{(\mathrm{I})}(t, f)-P_{x}^{(\mathrm{II})}(t, f)\right|}{\left\|S_{\boldsymbol{H}_{x}}\right\|_{1}^{2}} \leq \inf _{\alpha}\left\{B_{\alpha}\right\}
$$

where

$$
B_{\alpha} \triangleq m_{x}^{(\phi)}+2 m_{\boldsymbol{H}_{x}}^{(\tilde{\phi})}+2 \pi c_{\alpha} m_{\boldsymbol{H}_{x}}^{(0,1)} m_{\boldsymbol{H}_{x}}^{(1,0)}+2 \pi|\alpha| m_{\boldsymbol{H}_{x}}^{(1,1)}
$$

with

$$
\begin{aligned}
& \phi(\tau, \nu)=\left|1-S_{\boldsymbol{P}}^{(\alpha)}(\tau, \nu)\right| \\
& \tilde{\phi}(\tau, \nu)=\left|1-S_{\tilde{\boldsymbol{P}}}^{(\alpha)}(\tau, \nu)\right|
\end{aligned}
$$

and

$$
c_{\alpha} \triangleq\left|\alpha+\frac{1}{2}\right|+\left|\alpha-\frac{1}{2}\right| \text {. }
$$

Thus, if the weighted integrals and moments involved in $B_{\alpha}$ are small for some $\alpha$, we have

$$
P_{x}^{(\mathrm{I})}(t, f) \approx P_{x}^{(\mathrm{II})}(t, f) .
$$

Small $m_{x}^{(\phi)}$ requires $S_{\boldsymbol{P}}^{(\alpha)}(\tau, \nu) \approx 1$ on the effective support of $\left|\bar{A}_{x}(\tau, \nu)\right|$, and small $m_{\left.\boldsymbol{H}_{x}\right)}^{(\tilde{\phi})}$ requires $S_{\tilde{\boldsymbol{P}}}^{(\alpha)}(\tau, \nu) \approx 1$ on the effective support of $\left|S_{\boldsymbol{H}_{x}}(\tau, \nu)\right|$. These approximations are favored by small effective supports of $\left|\bar{A}_{x}(\tau, \nu)\right|$ and $\left|S_{\boldsymbol{H}_{x}}(\tau, \nu)\right|$. Similarly, both $m_{\boldsymbol{H}_{x}}^{(0,1)} m_{\boldsymbol{H}_{x}}^{(1,0)}$ and $m_{\boldsymbol{H}_{r}}^{(1,1)}$ are small if $\left|S_{\boldsymbol{H}_{x}}(\tau, \nu)\right|$ is effectively concentrated in a small rectangular region about the origin. We conclude that for underspread $x(t)$ and $\boldsymbol{H}_{x}$, type I and type II spectra yield effectively equal results. Some examples are shown in Fig. 3.

\section{APPROXIMATE KARHUNEN-LOÈVE EXPANSION}

In a mathematical sense, the Karhunen-Loève (KL) expansion [89], [107] can be viewed as an extension of the PSD to 
nonstationary processes. The correlation operator $\boldsymbol{R}_{x}$ of a nonstationary process $x(t)$ with finite mean energy has orthonormal eigenfunctions $u_{k}(t)$ and absolutely summable, nonnegative eigenvalues $\lambda_{k}$. The KL theorem [89], [107] states that $x(t)$ has a mean-square convergent expansion in terms of the eigenfunctions $u_{k}(t)$; furthermore, the expansion coefficients $\left\langle x, u_{k}\right\rangle$ are uncorrelated and have mean power $\lambda_{k}$

$$
\begin{aligned}
x(t) & =\sum_{k=1}^{\infty}\left\langle x, u_{k}\right\rangle u_{k}(t) \\
\mathrm{E}\left\{\left\langle x, u_{k}\right\rangle\left\langle x, u_{l}\right\rangle^{*}\right\} & =\lambda_{k} \delta_{k l} .
\end{aligned}
$$

The KL expansion features a double orthogonality: orthogonality of the basis functions $u_{k}(t)$, and (statistical) orthogonality of the random coefficients $\left\langle x, u_{k}\right\rangle$.

For a stationary process $x(t)$, the KL eigenfunctions are given by the complex sinusoids $e_{f}(t)=e^{j 2 \pi f t}$ and the KL eigenvalues are given by the PSD $P_{x}(f)$. Thus, the KL eigenfunctions are known a priori, they feature perfect frequency localization, and they are highly structured (i.e., related by frequency shifts); furthermore, the KL eigenvalues have a precise interpretation as a power spectrum. Unfortunately, all these properties are lost in the case of a general nonstationary process. However, we will now show that for an underspread process, Gabor frames [34], [35] provide a highly structured set of TF-localized approximate KL eigenfunctions, and both type I and type II TF spectra can be interpreted as an associated approximate KL eigenvalue spectrum. Our subsequent discussion extends previous results of Kozek [27], [28], [108], [109]. Furthermore, results in a similar spirit have been obtained in [67], [110] (using local cosine bases) and in [111], [112] (using wavelets).

\section{A. Approximate Decorrelation}

The "approximate KL expansion" we consider is given by the Gabor expansion [34], [35]

$$
x(t)=\sum_{k=-\infty}^{\infty} \sum_{l=-\infty}^{\infty}\left\langle x, u_{k, l}\right\rangle v_{k, l}(t) .
$$

Here, $\left\{u_{k, l}(t)\right\}$ and $\left\{v_{k, l}(t)\right\}(k, l \in \mathbb{Z})$ are dual Gabor frames for $L_{2}(\mathbb{R})$ [35], [113] that are obtained by TF shifting two "prototype" functions $u(t)$ and $v(t)$, respectively, according to

$$
u_{k, l}(t)=\left(\boldsymbol{S}_{k T, l F}^{(\alpha)} u\right)(t), v_{k, l}(t)=\left(\boldsymbol{S}_{k T, l F}^{(\alpha)} v\right)(t)
$$

(cf. (3)). Here, $T$ and $F$ are lattice constants satisfying $T F \leq 1$. We note that the Gabor frames $\left\{u_{k, l}(t)\right\}$ and $\left\{v_{k, l}(t)\right\}$ are dual iff $u(t)$ and $v(t)$ satisfy the biorthogonality condition [34], [35]

$$
\left\langle\boldsymbol{S}_{k / F, l / T}^{(\alpha)} u, \boldsymbol{S}_{k^{\prime} / F, l^{\prime} / T}^{(\alpha)} v\right\rangle=\delta_{k, k^{\prime}} \delta_{l, l^{\prime}}
$$

This relation replaces the deterministic orthogonality property of the KL expansion.

We consider oversampled $(T F<1)$ dual Gabor frames rather than an orthonormal Gabor basis. The reason is that an orthonormal Gabor basis presupposes critical sampling $(T F=$ 1 ), which according to the Balian-Low theorem implies poor TF localization of the Gabor basis functions [34], [35]. Under our assumptions, the expansion (30) holds for any signal $x(t) \in$ $L_{2}(\mathbb{R})$. The Gabor frames $\left\{u_{k, l}(t)\right\}$ and $\left\{v_{k, l}(t)\right\}$ are highly structured since their elements are related by TF shifts; moreover, the Gabor expansion can be efficiently implemented using the fast Fourier transform (FFT) [35].

In order to be able to interpret the Gabor expansion (30) as an approximate KL expansion, we desire that the expansion coefficients $\left\langle x, u_{k, l}\right\rangle$ be approximately statistically orthogonal, i.e.,

$$
\mathrm{E}\left\{\left\langle x, u_{k, l}\right\rangle\left\langle x, u_{k^{\prime}, l^{\prime}}\right\rangle^{*}\right\} \equiv\left\langle\boldsymbol{R}_{x} u_{k^{\prime}, l^{\prime}}, u_{k, l}\right\rangle \approx 0
$$

for $(k, l) \neq\left(k^{\prime}, l^{\prime}\right)$. This is analogous to the statistical orthogonality property in (29) and corresponds to an approximate diagonalization of the correlation operator $\boldsymbol{R}_{x}$. The associated approximation error can be bounded as follows.

Theorem 17: For any Gabor frame $\left\{u_{k, l}(t)\right\}$, the correlation of the coefficients $\left\langle x, u_{k, l}\right\rangle$ is bounded as

$$
\frac{\left|\mathrm{E}\left\{\left\langle x, u_{k, l}\right\rangle\left\langle x, u_{k^{\prime}, l^{\prime}}\right\rangle^{*}\right\}\right|}{\left\|\bar{A}_{x}\right\|_{1}} \leq m_{x}^{\left(\phi_{k-k^{\prime}, l-l^{\prime}}\right)}
$$

where $\phi_{k, l}(\tau, \nu)=\left|A_{u}(\tau+k T, \nu+l F)\right|$.

Thus, for underspread processes where $m_{x}^{\left(\phi_{k, l}\right)}$ can be made small for $(k, l) \neq(0,0)$ by a suitable choice of $u(t), T$, and $F$, the expansion coefficients $\left\langle x, u_{k, l}\right\rangle$ are approximately statistically orthogonal, i.e., the approximate decorrelation (31) holds. Small $m_{x}^{\left(\phi_{k, l}\right)}$ for $(k, l) \neq(0,0)$ requires that $\mid A_{u}(\tau+k T, \nu+$ $l F) \mid \approx 0$ for $(\tau, \nu) \in \mathcal{G}_{x}$, where $\mathcal{G}_{x}$ denotes the effective support of $\left|\bar{A}_{x}(\tau, \nu)\right|$. An equivalent formulation of this condition is $\left|A_{u}(\tau, \nu)\right| \approx 0$ for $(\tau, \nu) \in \tilde{\mathcal{G}}_{x}$, where $\tilde{\mathcal{G}}_{x}$ is $\mathcal{G}_{x}$ shifted by $(\tau, \nu)=(k T, l F)$. In order for this condition to be satisfied for all $(k, l) \neq(0,0),\left|A_{u}(\tau, \nu)\right|$ is required to decay quickly outside the effective support of $\left|\bar{A}_{x}(\tau, \nu)\right|$, which in turn is possible only if $x(t)$ is underspread and $u(t)$ is well TF localized.

\section{B. Approximate KL Eigenvalue Spectrum}

According to (29), the KL eigenvalues $\lambda_{k}$ are equal to the mean power of the expansion coefficients $\left\langle x, u_{k}\right\rangle$, i.e., $\mathrm{E}\left\{\left|\left\langle x, u_{k}\right\rangle\right|^{2}\right\}=\lambda_{k}$. In the stationary case, $\lambda_{k}$ becomes equal to the PSD $P_{x}(f)$. We will now show that for an underspread process $x(t)$, an "approximate KL eigenvalue spectrum" is provided by the samples of a type I or type II spectrum at the TF points $(t, f)=(k T, l F)$. Our development is based on the observation that the mean power of $\left\langle x, u_{k, l}\right\rangle$ equals the physical spectrum with window $u(t)$ at the TF points $(k T, l F)$, i.e.,

$$
\mathrm{E}\left\{\left|\left\langle x, u_{k, l}\right\rangle\right|^{2}\right\}=\operatorname{PS}_{x}^{(u)}(k T, l F) .
$$

The physical spectrum is a specific type I spectrum. Hence, the difference between $\mathrm{E}\left\{\left|\left\langle x, u_{k, l}\right\rangle\right|^{2}\right\}$ and a sampled type I spectrum $P_{x}^{(\mathrm{I})}(k T, l F)$ or a sampled type II spectrum $P_{x}^{(\mathrm{II})}(k T, l F)$ can be bounded by invoking Theorem 14 or Theorem 16, respectively.

Corollary 18: For any type I spectrum $P_{x}^{(\mathrm{I})}(t, f)=$ $\left\langle\boldsymbol{R}_{x}, \boldsymbol{P}_{t, f}\right\rangle$, we have

$$
\frac{\left|\mathrm{E}\left\{\left|\left\langle x, u_{k, l}\right\rangle\right|^{2}\right\}-P_{x}^{(\mathrm{I})}(k T, l F)\right|}{\left\|\bar{A}_{x}\right\|_{1}} \leq m_{x}^{\left(\phi_{1}\right)}
$$


where $\phi_{1}(\tau, \nu)=\left|A_{u}^{(\alpha)}(\tau, \nu)-S_{\boldsymbol{P}}^{(\alpha)}(\tau, \nu)\right|$. Furthermore, for any type II spectrum $P_{x}^{(\mathrm{II})}(t, f)=\left|\left\langle\boldsymbol{H}_{x}, \boldsymbol{P}_{t, f}\right\rangle\right|^{2}$

$$
\frac{\left|\mathrm{E}\left\{\left|\left\langle x, u_{k, l}\right\rangle\right|^{2}\right\}-P_{x}^{(\mathrm{II})}(k T, l F)\right|}{\left\|S_{\boldsymbol{H}_{x}}\right\|_{1}^{2}} \leq \inf _{\alpha}\left\{\beta_{\alpha}\right\} .
$$

Here

$$
\beta_{\alpha}=m_{x}^{\left(\phi_{2}\right)}+2 m_{\boldsymbol{H}_{x}}^{\left(\phi_{3}\right)}+2 \pi c_{\alpha} m_{\boldsymbol{H}_{x}}^{(0,1)} m_{\boldsymbol{H}_{x}}^{(1,0)}+2 \pi|\alpha| m_{\boldsymbol{H}_{x}}^{(1,1)}
$$

with

$$
\begin{aligned}
& \phi_{2}(\tau, \nu)=\left|1-A_{u}^{(\alpha)}(\tau, \nu)\right| \\
& \phi_{3}(\tau, \nu)=\left|1-S_{P}^{(\alpha)}(\tau, \nu)\right| .
\end{aligned}
$$

This corollary shows that for underspread processes where the bounds on the right-hand side of (32) and (33) can be made small (cf. the discussions following Theorems 14 and 16), one has

$$
\begin{aligned}
& \mathrm{E}\left\{\left|\left\langle x, u_{k, l}\right\rangle\right|^{2}\right\} \approx P_{x}^{(\mathrm{I})}(k T, l F) \\
& \mathrm{E}\left\{\left|\left\langle x, u_{k, l}\right\rangle\right|^{2}\right\} \approx P_{x}^{(\mathrm{II})}(k T, l F) .
\end{aligned}
$$

Thus, the sampled TF spectra $P_{x}^{(\mathrm{I})}(k T, l F)$ and $P_{x}^{(\mathrm{II})}(k T, l F)$ constitute an approximate KL eigenvalue spectrum. Note that for $P_{x}^{(\mathrm{I})}(t, f)=\operatorname{PS}_{x}^{(u)}(t, f)$, the left-hand approximation in (34) becomes exact.

For underspread processes, one can furthermore show the approximate eigenvalue relation [83]

$$
\left(\boldsymbol{R}_{x} u_{k, l}\right)(t) \approx P_{x}^{(\mathrm{I})}(k T, l F) u_{k, l}(t) \approx P_{x}^{(\mathrm{II})}(k T, l F) u_{k, l}(t) .
$$

\section{INPUT-OUTPUT RELATIONS}

The action of a linear time-invariant system $\boldsymbol{K}$ with impulse response $k(\tau)$ on the second-order statistics of a stationary process is described in a simple way by the PSD: for $y(t)=(\boldsymbol{K} x)(t)=(k * x)(t)$, we have

$$
\begin{aligned}
P_{y}(f) & =|K(f)|^{2} P_{x}(f) \\
P_{y x}(f) & =K(f) P_{x}(f) \\
P_{x y}(f) & =K^{*}(f) P_{x}(f)
\end{aligned}
$$

where $K(f)=\int_{\tau} k(\tau) e^{-j 2 \pi f \tau} d \tau$ is the frequency response of $\boldsymbol{K}$. These multiplicative input-output relations for the PSD can be extended in an approximate manner to the case where an underspread nonstationary process is passed through an underspread LTV system [83]. The resulting approximate input-output relations are useful for the design of LTV filters [42], [47], [114], [115]. While we will here consider only the GWVS and GES, approximate input-output relations hold also for other type I and II spectra due to the approximate equivalence of TF spectra in the underspread case (see Section VI). We note that input-output relations for nonstationary processes have previously been considered in [8], [116], however only for time-invariant systems.

\section{A. Input-Output Relations for the Generalized Wigner-Ville Spectrum}

We consider an LTV system $\boldsymbol{K}$ whose input is a nonstationary process $x(t)$. The system output $y(t)=(\boldsymbol{K} x)(t)$ is a nonstationary process whose correlation operator is given by $\boldsymbol{R}_{y}=\boldsymbol{K} \boldsymbol{R}_{x} \boldsymbol{K}^{+}$. Furthermore, $\boldsymbol{R}_{y x}=\boldsymbol{K} \boldsymbol{R}_{x}$ and $\boldsymbol{R}_{x y}=\boldsymbol{R}_{x} \boldsymbol{K}^{+}$. An approximate TF formulation of these input-output relations in terms of the GWVS of $x(t)$ and the GWS of $K$ can be based on the next result.

Theorem 19: Let $y(t)=(\boldsymbol{K} x)(t)$. The differences

$$
\begin{aligned}
& \Delta_{1}(t, f) \triangleq \bar{W}_{y}^{(\alpha)}(t, f)-\left|L_{\boldsymbol{K}}^{(\alpha)}(t, f)\right|^{2} \bar{W}_{x}^{(\alpha)}(t, f) \\
& \Delta_{2}(t, f) \triangleq \bar{W}_{y x}^{(\alpha)}(t, f)-L_{\boldsymbol{K}}^{(\alpha)}(t, f) \bar{W}_{x}^{(\alpha)}(t, f) \\
& \Delta_{3}(t, f) \triangleq \bar{W}_{x y}^{(\alpha)}(t, f)-L_{\boldsymbol{K}}^{(\alpha) *}(t, f) \bar{W}_{x}^{(\alpha)}(t, f)
\end{aligned}
$$

are bounded as

$$
\begin{aligned}
\frac{\left|\Delta_{1}(t, f)\right|}{\left\|S_{\boldsymbol{K}}\right\|_{1}^{2}\left\|\bar{A}_{x}\right\|_{1}} \leq & 4 \pi|\alpha| m_{\boldsymbol{K}}^{(1,1)}+2 \pi c_{\alpha}\left[m_{\boldsymbol{K}}^{(0,1)} m_{x}^{(1,0)}\right. \\
& \left.+m_{\boldsymbol{K}}^{(1,0)} m_{x}^{(0,1)}+m_{\boldsymbol{K}}^{(0,1)} m_{\boldsymbol{K}}^{(1,0)}\right] \\
\frac{\left|\Delta_{2}(t, f)\right|}{\left\|S_{\boldsymbol{K}}\right\|_{1}\left\|\bar{A}_{x}\right\|_{1} \leq} & 2 \pi\left[\left|\alpha+\frac{1}{2}\right| m_{\boldsymbol{K}}^{(1,0)} m_{x}^{(0,1)}\right. \\
& \left.+\left|\alpha-\frac{1}{2}\right| m_{\boldsymbol{K}}^{(0,1)} m_{x}^{(1,0)}\right] \\
\frac{\left|\Delta_{3}(t, f)\right|}{\left\|S_{\boldsymbol{K}}\right\|_{1}\left\|\bar{A}_{x}\right\|_{1} \leq} & 2 \pi\left[\left|\alpha+\frac{1}{2}\right| m_{\boldsymbol{K}}^{(0,1)} m_{x}^{(1,0)}\right. \\
& \left.+\left|\alpha-\frac{1}{2}\right| m_{\boldsymbol{K}}^{(1,0)} m_{x}^{(0,1)}+2|\alpha| m_{\boldsymbol{K}}^{(1,1)}\right] .
\end{aligned}
$$

The above bounds are small if the effective supports of the GEAF $\left|\bar{A}_{x}(\tau, \nu)\right|$ and the GSF $\left|S_{K}(\tau, \nu)\right|$ are both contained within a small rectangular region about the origin (i.e., if $x(t)$ and $\boldsymbol{K}$ are "jointly underspread" [22], [30], [83]). In that case, the theorem yields the approximations

$$
\begin{aligned}
& \bar{W}_{y}^{(\alpha)}(t, f) \approx\left|L_{\boldsymbol{K}}^{(\alpha)}(t, f)\right|^{2} \bar{W}_{x}^{(\alpha)}(t, f) \\
& \bar{W}_{y x}^{(\alpha)}(t, f) \approx L_{\boldsymbol{K}}^{(\alpha)}(t, f) \bar{W}_{x}^{(\alpha)}(t, f) \\
& \bar{W}_{x y}^{(\alpha)}(t, f) \approx L_{\boldsymbol{K}}^{(\alpha) *}(t, f) \bar{W}_{x}^{(\alpha)}(t, f) .
\end{aligned}
$$

The bounds in (35) and (36) are strongest for $\alpha=0$ since here $L_{\boldsymbol{K}^{+}}^{(0)}(t, f)=L_{\boldsymbol{K}}^{(0) *}(t, f)$ (note, however, that for $\boldsymbol{K}$ underspread we have $L_{\boldsymbol{K}^{+}}^{(0)}(t, f) \approx L_{\boldsymbol{K}}^{(0) *}(t, f)$ even for $\alpha \neq 0$ [30], [83]). The approximation (37) is illustrated for $\alpha=0$ in Fig. 5.

\section{B. Input-Output Relations for the Generalized Evolutionary Spectrum}

Next, we consider an alternative approximate TF formulation of the relation $\boldsymbol{R}_{y}=\boldsymbol{K} \boldsymbol{R}_{x} \boldsymbol{K}^{+}$in terms of the GES of $x(t)$ and the GWS of $\boldsymbol{K}$. We shall use the fact that if $\boldsymbol{H}_{x}$ is an innovations system for $x(t)$, then $\boldsymbol{H}_{y}=\boldsymbol{K} \boldsymbol{H}_{x}$ is an innovations system for $y(t)=(\boldsymbol{K} x)(t)$ (indeed, $x(t)=\left(\boldsymbol{H}_{x} n\right)(t)$ entails 


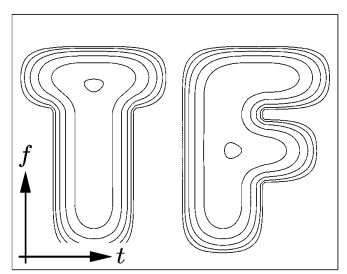

(a)

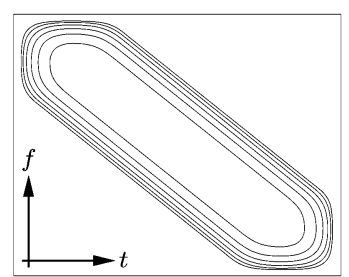

(b)

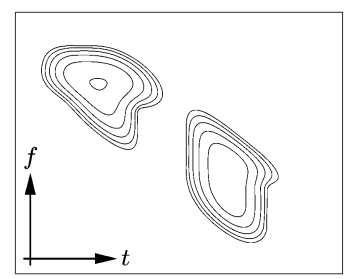

(c)

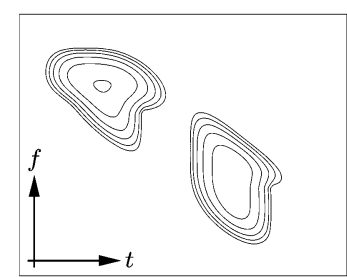

(d)

Fig. 5. Illustration of the approximate input-output relation (37) for the Wigner-Ville spectrum (GWVS with $\alpha=0)$. (a) Wigner-Ville spectrum of input process $x(t)$, (b) Weyl symbol of LTV system $\boldsymbol{K}$, (c) Wigner-Ville spectrum of output process $y(t)=(\boldsymbol{K} x)(t),(\mathrm{d})$ approximation $\left|L_{\boldsymbol{K}}^{(0)}(t, f)\right|^{2} \bar{W}_{x}^{(0)}(t, f)$.

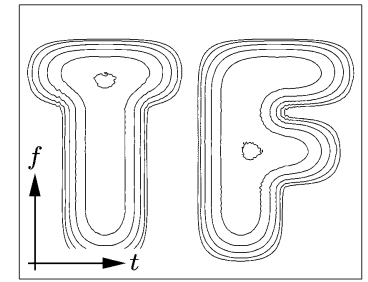

(a)

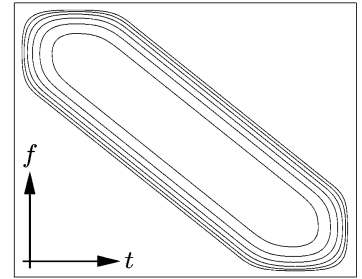

(b)

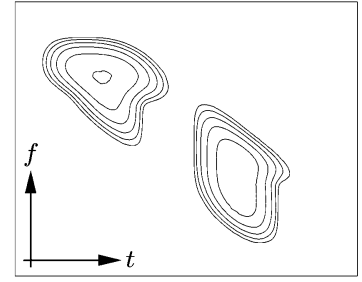

(c)

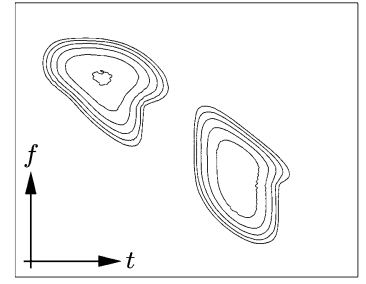

(d)

Fig. 6. Illustration of the approximate input-output relation (38) for the Weyl spectrum (GES with $\alpha=0)$. (a) Weyl spectrum of input process $x(t)$, (b) Weyl symbol of LTV system $\boldsymbol{K}$, (c) Weyl spectrum of output process $y(t)=(\boldsymbol{K} x)(t)$, (d) approximation $\left|L_{\boldsymbol{K}}^{(0)}(t, f)\right|^{2} G_{x}^{(0)}(t, f)$.

$\left.y(t)=\left(K \boldsymbol{H}_{x} n\right)(t)\right)$. Thus, in the following we assume that the GES of $x(t)$ uses the innovations system $\boldsymbol{H}_{x}$ and the GES of $y(t)=(\boldsymbol{K} x)(t)$ uses the innovations system $\boldsymbol{K} \boldsymbol{H}_{x}$. If $\boldsymbol{H}_{x}$ and $\boldsymbol{K}$ are causal, then $\boldsymbol{K} \boldsymbol{H}_{x}$ is causal as well. However, if $\boldsymbol{H}_{x}$ is the positive semidefinite square root of $\boldsymbol{R}_{x}$, i.e., $\boldsymbol{H}_{x}=\boldsymbol{R}_{x}^{1 / 2}$, then $\boldsymbol{K} \boldsymbol{H}_{x}$ is not the positive semidefinite square root of $\boldsymbol{R}_{y}$ unless $\boldsymbol{K}$ is positive semidefinite and $\boldsymbol{K}$ and $\boldsymbol{H}_{x}$ commute. (The latter condition is approximately satisfied if $\boldsymbol{K}$ and $\boldsymbol{H}_{x}$ are jointly underspread [30], [83].)

Theorem 20: Let $y(t)=(\boldsymbol{K} x)(t)$ and

$$
\begin{aligned}
& G_{x}^{(\alpha)}(t, f)=\left|L_{\boldsymbol{H}_{x}}^{(\alpha)}(t, f)\right|^{2} \\
& G_{y}^{(\alpha)}(t, f)=\left|L_{\boldsymbol{K} \boldsymbol{H}_{x}}^{(\alpha)}(t, f)\right|^{2} .
\end{aligned}
$$

Then, the difference

$$
\Delta(t, f) \triangleq G_{y}^{(\alpha)}(t, f)-\left|L_{K}^{(\alpha)}(t, f)\right|^{2} G_{x}^{(\alpha)}(t, f)
$$

is bounded as

$$
\frac{|\Delta(t, f)|}{\left\|S_{\boldsymbol{K}}\right\|_{1}^{2}\left\|S_{\boldsymbol{H}_{x}}\right\|_{1}^{2}} \leq 4 \pi c_{\alpha}\left[m_{\boldsymbol{K}}^{(0,1)} m_{\boldsymbol{H}_{x}}^{(1,0)}+m_{\boldsymbol{K}}^{(1,0)} m_{\boldsymbol{H}_{x}}^{(0,1)}\right] .
$$

Thus, if $m_{\boldsymbol{K}}^{(0,1)} m_{\boldsymbol{H}_{x}}^{(1,0)}+m_{\boldsymbol{K}}^{(1,0)} m_{\boldsymbol{H}_{x}}^{(0,1)}$ is small, then

$$
G_{y}^{(\alpha)}(t, f) \approx\left|L_{K}^{(\alpha)}(t, f)\right|^{2} G_{x}^{(\alpha)}(t, f) .
$$

Small $m_{\boldsymbol{K}}^{(0,1)} m_{\boldsymbol{H}_{x}}^{(1,0)}+m_{\boldsymbol{K}}^{(1,0)} m_{\boldsymbol{H}_{r}}^{(0,1)}$ requires that the effective supports of $\left|S_{\boldsymbol{H}_{x}}(\tau, \nu)\right|$ and $\left|S_{\boldsymbol{K}}(\tau, \nu)\right|$ are both contained in a small rectangle about the origin, i.e., $\boldsymbol{H}_{x}$ and $\boldsymbol{K}$ are jointly underspread [30], [83]. This implies that $x(t)$ and $\boldsymbol{K}$ are jointly underspread. The approximation (38) is shown in Fig. 6 for $\alpha=0, \boldsymbol{H}_{x}=\boldsymbol{R}_{x}^{1 / 2}$, and $\boldsymbol{H}_{y}=\boldsymbol{K} \boldsymbol{R}_{x}^{1 / 2}$. Note that because $x(t)$ and $\boldsymbol{K}$ are jointly underspread, also $y(t)$ is underspread and hence the Weyl spectrum of $y(t)$ in Fig. 6(c) is practically equal to the Wigner-Ville spectrum of $y(t)$ in Fig. 5(c).

\section{CONCLUSION}

The desire to extend the power spectral density to nonstationary random processes has led to the definition of a rich-if somewhat confusing - variety of different time-dependent or time-frequency (TF) spectra for nonstationary processes. In this paper, we introduced a unifying framework for the known class of type I TF spectra and the new class of type II TF spectra. This framework, which encompasses all major TF spectra defined so far, is based on TF operator symbols induced by certain "prototype operators."

We then used this unifying formulation for a profound study of the properties of type I and type II spectra in the practically important case of underspread processes (i.e., nonstationary processes with small $\mathrm{TF}$ correlation spread). In particular, we stated a number of fundamental approximations for type I and type II spectra of underspread processes. These approximations show that in the underspread case

- all type I and type II spectra satisfy several desirable properties (marginals, positivity, etc.) at least in an approximate manner;

- different type I and type II spectra yield effectively identical results; this also implies that all type I and type II spectra are "almost complete" second-order statistics of an underspread process;

- all type I and type II spectra are smooth 2-D lowpass functions;

- Gabor frames provide an approximate Karhunen-Loève (KL) expansion, and type I and type II spectra provide an approximate KL eigenvalue spectrum;

- the effect of underspread linear time-varying systems can be described in the TF domain by simple approximate input-output relations for type I and type II spectra.

We substantiated these approximations mathematically by providing upper bounds on the associated approximation errors. 
These bounds are formulated in terms of weighted integrals and moments of the expected ambiguity function of the process and/or of the spreading function of an innovations system; they can usually be reliably estimated in practice.

For overspread processes, none of the above approximations is valid. Here, the results obtained with different type I or type II spectra may differ dramatically. Nonsmoothed TF spectra such as the Wigner-Ville spectrum and the evolutionary spectrum contain large statistical cross terms. These cross terms indicate the strong high-lag TF correlations of an overspread process but, on the other hand, impede a clear representation of the process' mean TF energy distribution. In contrast, in smoothed TF spectra such as the (type I or type II) physical spectrum, statistical cross terms are attenuated or suppressed. Thus, these spectra do not correctly indicate the process' TF correlations; on the other hand, they often do provide a reasonably faithful picture of the process' mean TF energy distribution even in the overspread case. Finally, in the overspread case, many TF spectra do not satisfy numerous desirable properties even approximately, and they cannot be used to formulate simple input-output relations in the TF domain.

We can thus conclude that the concept of a TF spectrum is most successful and satisfactory in the underspread case. For underspread processes, our results indicate that the choice of a specific TF spectrum is not critical because all major existing TF spectra produce meaningful results and satisfy a number of desirable properties at least approximately. Our approximations show that type I and II TF spectra provide tools for the secondorder analysis and linear filtering of underspread processes that are just as powerful, intuitive, and practically convenient as the power spectral density of stationary processes.

\section{ACKNOWLEDGMENT}

The authors would like to thank the anonymous reviewers whose comments have resulted in an improvement of this paper.

\section{REFERENCES}

[1] C. H. Page, "Instantaneous power spectra," J. Appl. Phys, vol. 23, pp. 103-106, Jan. 1952.

[2] D. G. Lampard, "Generalization of the Wiener-Khintchine theorem to nonstationary processes," J. Appl. Phys, vol. 25, pp. 802-803, Jun. 1954.

[3] M. J. Levin, "Instantaneous spectra and ambiguity functions," IEEE Trans. Inf. Theory, vol. IT-10, no. 1, pp. 95-97, Jan. 1964.

[4] M. R. Dubman, "The Spectral Characterization and Comparison of NonStationary Processes,' Unpublished Rocketdyne Research Report, 1965.

[5] M. B. Priestley, "Evolutionary spectra and nonstationary processes," $J$. Roy. Statist. Soc. Ser. B, vol. 27, no. 2, pp. 204-237, 1965.

[6] R. M. Loynes, "On the concept of the spectrum for non-stationary processes," J. Roy. Statist. Soc. Ser. B, vol. 30, no. 1, pp. 1-30, 1968.

[7] A. W. Rihaczek, "Signal energy distribution in time and frequency," IEEE Trans. Inf. Theory, vol. IT-14, no. 3, pp. 369-374, May 1968.

[8] W. D. Mark, "Spectral analysis of the convolution and filtering of nonstationary stochastic processes," J. Sound Vib., vol. 11, no. 1, pp. 19-63, 1970.

[9] M. B. Priestley, "Some notes on the physical interpretation of spectra of nonstationary stochastic processes," J. Sound Vib., vol. 17, pp. 51-54, 1971.

[10] D. Tjøstheim, "Spectral generating operators for nonstationary processes," Adv. Appl. Probab., vol. 8, pp. 831-846, 1976.

[11] G. Mélard, "Propriétés du spectre évolutif d'un processus nonstationnaire," Ann. Inst. H. Poincaré B, vol. XIV, no. 4, pp. 411-424, 1978.

[12] F. Battaglia, "Some extensions of the evolutionary spectral analysis of a stochastic process," Bull. Unione Mat. Italiana, vol. 16-B, no. 5, pp. 1154-1166, 1979.
[13] O. D. Grace, "Instantaneous power spectra," J. Acoust. Soc. Amer, vol. 69, no. 1, pp. 191-198, 1981.

[14] W. Martin and P. Flandrin, "Wigner-Ville spectral analysis of nonstationary processes," IEEE Trans. Acoust., Speech, Signal Process., vol. ASSP-33, no. 6, pp. 1461-1470, Dec. 1985.

[15] G. Mélard and A. de Schutter-Herteleer, "Contributions to evolutionary spectral theory,” J. Roy. Statist. Soc. Ser. B, vol. 10, pp. 41-63, 1989.

[16] P. Flandrin, "Time-dependent spectra for nonstationary stochastic processes," in Time and Frequency Representation of Signals and Systems, G. Longo and B. Picinbono, Eds. Wien, Austria: Springer, 1989, pp. 69-124.

[17] R. D. Hippenstiel and P. M. De Oliveira, "Time-varying spectral estimation using the instantaneous power spectrum (IPS)," IEEE Trans. Acoust., Speech, Signal Process., vol. 38, no. 10, pp. 1752-1759, Oct. 1990.

[18] I. G. Zurbenko, "Spectral analysis of non-stationary time series," Int. Statist. Rev., vol. 59, no. 2, pp. 163-173, 1991.

[19] M. Amin, "Time-frequency spectrum analysis and estimation for nonstationary random processes," in Advances in Spectrum Estimation, B. Boashash, Ed. Melbourne, Australia: Longman Cheshire, 1992, pp. 208-232.

[20] C. S. Detka and A. El-Jaroudi, "The transitory evolutionary spectrum," in Proc. IEEE Int. Conf. Acoustics, Speech, and Signal Processing (ICASSP-94), Adelaide, Australia, Apr. 1994, pp. 289-292.

[21] J. A. Sills, "Nonstationary Signal Modeling, Filtering, and Parameterization,” Ph.D. dissertation, Georgia Inst. Technol., Atlanta, GA, Mar. 1995.

[22] G. Matz, F. Hlawatsch, and W. Kozek, "Generalized evolutionary spectral analysis and the Weyl spectrum of nonstationary random processes," IEEE Trans. Signal Process., vol. 45, no. 6, pp. 1520-1534, Jun. 1997.

[23] P. Flandrin and W. Martin, "The Wigner-Ville spectrum of nonstationary random signals," in The Wigner Distribution-Theory and Applications in Signal Processing, W. Mecklenbräuker and F. Hlawatsch, Eds. Amsterdam, The Netherlands: Elsevier, 1997, pp. 211-267.

[24] P. Flandrin, Time-Frequency/Time-Scale Analysis. San Diego, CA: Academic, 1999.

[25] W. Kozek, F. Hlawatsch, H. Kirchauer, and U. Trautwein, "Correlative time-frequency analysis and classification of nonstationary random processes," in Proc. IEEE-SP Int. Symp. Time-Frequency Time-Scale Analysis, Philadelphia, PA, Oct. 1994, pp. 417-420.

[26] W. Kozek, "On the underspread/overspread classification of nonstationary random processes," in Proc. Int. Conf. Industrial and Applied Mathematics, K. Kirchgässner, O. Mahrenholtz, and R. Mennicken, Eds. Berlin, Germany: Akademie-Verlag, 1996, pp. 63-66. Vol. 3 of Mathematical Research.

[27] — , "Matched Weyl-Heisenberg Expansions of Nonstationary Environments," Ph.D. dissertation, Vienna Univ. Technol., Vienna, Austria, Mar. 1997.

[28] - "Adaptation of Weyl-Heisenberg frames to underspread environments," in Gabor Analysis and Algorithms: Theory and Applications, $\mathrm{H}$. G. Feichtinger and T. Strohmer, Eds. Boston, MA: Birkhäuser, 1998, ch. 10 , pp. 323-352.

[29] G. Matz and F. Hlawatsch, "Time-varying spectra for underspread and overspread nonstationary processes," in Proc. 32nd Asilomar Conf. Signals, Systems, Computers, Pacific Grove, CA, Nov. 1998, pp. 282-286.

[30] G. Matz, "A Time-Frequency Calculus for Time-Varying Systems and Nonstationary Processes with Applications," Ph.D. dissertation, Vienna Univ. Technology, Vienna, Austria, Nov. 2000.

[31] G. Matz and F. Hlawatsch, "Time-varying power spectra of nonstationary random processes," in Time-Frequency Signal Analysis and Processing: A Comprehensive Reference, B. Boashash, Ed. Oxford, U.K.: Elsevier, 2003, pp. 400-409.

[32] F. Hlawatsch and G. Matz, "Temps-fréquence et traitement statistique," in Temps-fréquence: concepts et outils, F. Hlawatsch and F. Auger, Eds. Paris, France: Hermes/Lavoisier, 2005, pp. 289-330.

[33] R. Thomä, J. Steffens, and U. Trautwein, "Statistical cross-terms in quadratic time-frequency distributions," in Proc. Int. Conf. Digital Signal Processing, Nicosia, Cyprus, Jul. 1993, pp. 88-99.

[34] K. Gröchenig, Foundations of Time-Frequency Analysis. Boston, MA: Birkhäuser, 2001.

[35] H. G. Feichtinger and T. Strohmer, Eds., Gabor Analysis and Algorithms: Theory and Applications. Boston, MA: Birkhäuser, 1998.

[36] N. A. Abdrabbo and M. B. Priestley, "Filtering non-stationary signals," J. Roy. Statist. Soc. Ser. B, vol. 31, pp. 150-159, 1969.

[37] P. Flandrin, "A time-frequency formulation of optimum detection," IEEE Trans. Acoust., Speech, Signal Process., vol. 36, no. 9, pp. 1377-1384, Sep. 1988. 
[38] I. Vincent, C. Doncarli, and E. Le Carpentier, "Nonstationary signals classification using time-frequency distributions," in Proc. IEEE-SP Int. Symp. Time-Frequency Time-Scale Analysis, Philadelphia, PA, Oct. 1994, pp. 233-236.

[39] A. M. Sayeed, P. Lander, and D. L. Jones, "Improved time-frequency filtering of signal-averaged electrocardiograms," J. Electrocardiol., vol. 28, pp. 53-58, 1995.

[40] A. M. Sayeed and D. L. Jones, "Optimal detection using bilinear timefrequency and time-scale representations," IEEE Trans. Signal Process., vol. 43, no. 12, pp. 2872-2883, Dec. 1995.

[41] J. A. Sills and E. W. Kamen, "Wiener filtering of nonstationary signals based on spectral density functions," in Proc. 34th IEEE Conf. Decision and Control, Kobe, Japan, Dec. 1995, pp. 2521-2526.

[42] G. Matz and F. Hlawatsch, "Time-frequency formulation and design of optimal detectors," in Proc. IEEE-SP Int. Symp. Time-Frequency TimeScale Analysis, Paris, France, Jun. 1996, pp. 213-216.

[43] H. A. Khan and L. F. Chaparro, "Nonstationary Wiener filtering based on evolutionary spectral theory," in Proc. IEEE Int. Conf. Acoustics, Speech, and Signal Processing (ICASSP-97), Munich, Germany, May 1997, pp. 3677-3680.

[44] G. Matz and F. Hlawatsch, "Time-frequency methods for signal detection with application to the detection of knock in car engines," in Proc. IEEE-SP Workshop on Statistical Signal and Array Processing, Portland, OR, Sep. 1998, pp. 196-199.

[45] F. Hlawatsch, Time-Frequency Analysis and Synthesis of Linear Signal Spaces: Time-Frequency Filters, Signal Detection and Estimation, and Range-Doppler Estimation. Boston, MA: Kluwer, 1998.

[46] G. Matz and F. Hlawatsch, "Time-frequency subspace detectors and application to knock detection," Int. J. Electron. Commun. (AË̈), vol. 53, no. 6, pp. 379-385, 1999.

[47] F. Hlawatsch, G. Matz, H. Kirchauer, and W. Kozek, "Time-frequency formulation, design, and implementation of time-varying optimal filters for signal estimation," IEEE Trans. Signal Process., vol. 48, pp. 1417-1432, May 2000.

[48] G. Matz and F. Hlawatsch, "Time-frequency coherence analysis of nonstationary random processes," in Proc. IEEE-SP Workshop on Statistical Signal and Array Processing, Pocono Manor, PA, Aug. 2000, pp. 554-558.

[49] M. Davy, C. Doncarli, and G. F. Boudreaux-Bartels, "Improved optimization of time-frequency-based signal classifiers," IEEE Signal Process. Lett., vol. 8, pp. 52-57, Feb. 2001.

[50] F. Hlawatsch and G. Matz, "Time-frequency methods for signal estimation and detection," in Time-Frequency Signal Analysis and Processing: A Comprehensive Reference, B. Boashash, Ed. Oxford, U.K.: Elsevier, 2003, ch. 12.4 , pp. 528-537.

[51] Y. Grenier, "Time-dependent ARMA modeling of nonstationary signals," IEEE Trans. Acoust., Speech, Signal Process., vol. ASSP-31, no. 4, pp. 899-911, Aug. 1983.

[52] - "Parametric time-frequency representations," in Traitement $d u$ Signal/Signal Processing, Les Houches, Session XLV, J. L. Lacoume, T. S. Durrani, and R. Stora, Eds. Amsterdam, The Netherlands: Elsevier, 1987, pp. 338-397.

[53] M. Niedźwiecki, Identification of Time-Varying Processes. New York: Wiley, 2000.

[54] J. D. Thatcher and M. G. Amin, "The running bispectrum," in Proc. IEEE SP Int. Workshop Higher-Order Spectral Analysis, Vail, CO, Jun. 1989, pp. 36-40.

[55] J. R. Fonollosa and C. L. Nikias, "General class of time-frequency higher-order spectra: Definitions, properties, computation and applications to transient signals," in Proc. IEEE-SP Int. Workshop Higher-Order Statistics, Chamrousse, France, Jul. 1991, pp. 132-135.

[56] G. B. Giannakis and A. Dandawate, "Polyspectral analysis of nonstationary signals: Bases, consistency and HOS-WV," in Proc. IEEE-SP Int. Workshop Higher-Order Statistics, Chamrousse, France, Jul. 1991, pp. $167-170$.

[57] B. Boashash and P. Shea, "Polynomial Wigner-Ville distributions and their relationship to time-varying higher order spectra," IEEE Trans. Signal Process., vol. 42, no. 1, pp. 216-220, Jan. 1994.

[58] A. Hanssen and L. L. Scharf, "A theory of polyspectra for nonstationary stochastic processes," IEEE Trans. Signal Process., vol. 51, no. 5, pp. 1243-1252, May 2003.

[59] A. S. Kayhan, L. F. Chaparro, and A. El-Jaroudi, "Wold-Cramer evolutionary spectral estimators," in Proc. IEEE-SP Int. Symp. Time-Frequency Time-Scale Analysis, Victoria, BC, Canada, Oct. 1992, pp. $115-118$.
[60] W. Kozek and K. Riedel, "Quadratic time-varying spectral estimation for underspread processes," in Proc. IEEE-SP Int. Symp. Time-Frequency Time-Scale Analysis, Philadelphia, PA, Oct. 1994, pp. 460-463.

[61] K. Riedel, "Optimal kernel estimation of the instantaneous frequency," IEEE Trans. Signal Process., vol. 42, no. 10, pp. 2644-2649, Oct. 1994

[62] A. M. Sayeed and D. L. Jones, "Optimal kernels for nonstationary spectral estimation," IEEE Trans. Signal Process., vol. 43, no. 2, pp. 478-491, Feb. 1995

[63] S. B. Hearon and M. G. Amin, "Minimum-variance time-frequency distribution kernels," IEEE Trans. Signal Process., vol. 43, no. 5, pp. 1258-1262, May 1995.

[64] M. Bayram and R. Baraniuk, "Multiple window time-frequency analysis," in Proc. IEEE-SP Int. Symp. Time-Frequency Time-Scale Analysis, Paris, France, Jun. 1996, pp. 173-176.

[65] M. G. Amin, "Recursive kernels for time-frequency signal representations," IEEE Signal Process. Lett., vol. 3, no. 1, pp. 16-18, Jan. 1996.

[66] D. J. Thomson, "Multi-window spectrum estimation for non-stationary data," in Proc. IEEE-SP Workshop Statistical Signal and Array Proc, Portland, OR, Sep. 1998, pp. 344-347.

[67] S. G. Mallat, G. Papanicolaou, and Z. Zhang, "Adaptive covariance estimation of locally stationary processes," Ann. Statist., vol. 26, pp. 1-47, Feb. 1998.

[68] L. L. Scharf and B. Friedlander, "Toeplitz and Hankel kernels for estimating time-varying spectra of discrete-time random processes," IEEE Trans. Signal Process., vol. 49, no. 1, pp. 179-189, Jan. 2001.

[69] A. W. Naylor and G. R. Sell, Linear Operator Theory in Engineering and Science, 2nd ed. New York: Springer-Verlag, 1982.

[70] I. C. Gohberg and M. G. Krein, Introduction to the Theory of Linear Non-Selfadjoint Operators. Providence, RI: Amer. Math. Soc., 1969.

[71] K. E. Cahill and R. J. Glauber, "Ordered expansions in boson amplitude operators," Phys. Rev., vol. 177, pp. 1857-1881, Jan. 1969.

[72] L. Cohen, "Quantization problem and variational principle in the phasespace formulation of quantum mechanics," J. Math. Phys., vol. 17, pp. 1863-1866, Oct. 1976

[73] P. Flandrin, B. Escudié, and J. Gréa, "Règles de correspondance et $\star-$ produits dans le plan temps-fréquence," C. R. Acad. Sci. Paris, Sér. I, vol. 294, pp. 281-284, Feb. 1982.

[74] B.-G. Iem, A. Papandreou-Suppappola, and G. F. Boudreaux-Bartels, "Classes of smoothed Weyl symbols," IEEE Signal Process. Lett., vol. 7, no. 7, pp. 186-188, Jul. 2000.

[75] N. G. de Bruijn, "A theory of generalized functions, with applications to Wigner distribution and Weyl correspondence," Nieuw Archief voor Wiskunde, vol. XXI, pp. 205-280, 1973.

[76] G. B. Folland, Harmonic Analysis in Phase Space, vol. 122 of Annals of Mathematics Studies. Princeton, NJ: Princeton Univ. Press, 1989.

[77] W. Kozek, "Time-frequency signal processing based on the WignerWeyl framework," Signal Process., vol. 29, pp. 77-92, Oct. 1992.

[78] - "On the generalized Weyl correspondence and its application to time-frequency analysis of linear time-varying systems," in Proc. IEEE-SP Int. Symp. Time-Frequency Time-Scale Analysis, Victoria, BC, Canada, Oct. 1992, pp. 167-170.

[79] J. Ramanathan and P. Topiwala, "Time-frequency localization via the Weyl correspondence," SIAM J. Matrix Anal. Appl., vol. 24, no. 5, pp. 1378-1393, 1993.

[80] R. G. Shenoy and T. W. Parks, "The Weyl correspondence and timefrequency analysis," IEEE Trans. Signal Process., vol. 42, no. 2, pp. 318-331, Feb. 1994

[81] W. Kozek, "On the transfer function calculus for underspread LTV channels," IEEE Trans. Signal Process., vol. 45, no. 1, pp. 219-223, Jan. 1997.

[82] M. W. Wong, Weyl Transforms. New York: Springer-Verlag, 1998.

[83] G. Matz and F. Hlawatsch, "Time-frequency transfer function calculus (symbolic calculus) of linear time-varying systems (linear operators) based on a generalized underspread theory," J. Math. Phys. (Special Issue on Wavelet and Time-Frequency Analysis), vol. 39, pp. 4041-4071, Aug. 1998

[84] I. Daubechies, "Time-frequency localization operators: A geometric phase space approach," IEEE Trans. Inf. Theory, vol. 34, no. 4, pp. 605-612, Jul. 1988.

[85] - "The wavelet transform, time-frequency localization and signal analysis," IEEE Trans. Inf. Theory, vol. 36, no. 5, pp. 961-1005, Sep. 1990.

[86] J. Ramanathan and P. Topiwala, "Time-frequency localization and the spectrogram," Appl. Comput. Harm. Anal., vol. 1, pp. 209-215, 1994.

[87] F. Hlawatsch and W. Kozek, "Time-frequency projection filters and time-frequency signal expansions," IEEE Trans. Signal Process., vol. 42, no. 12, pp. 3321-3334, Dec. 1994. 
[88] S. M. Kay, Modern Spectral Estimation. Englewood Cliffs, NJ: Prentice-Hall, 1988.

[89] A. Papoulis, Probability, Random Variables, and Stochastic Processes, 3rd ed. New York: McGraw-Hill, 1991.

[90] L. Cohen, Time-Frequency Analysis. Englewood Cliffs, NJ: PrenticeHall, 1995.

[91] F. Hlawatsch and G. F. Boudreaux-Bartels, "Linear and quadratic timefrequency signal representations," IEEE Signal Process. Mag., vol. 9, no. 4, pp. 21-67, Apr. 1992.

[92] L. A. Zadeh, "Frequency analysis of variable networks," Proc. IRE, vol. 38, pp. 291-299, Mar. 1950.

[93] P. A. Bello, "Characterization of randomly time-variant linear channels," IEEE Trans. Commun. Syst., vol. COM-11, no. 4, pp. 360-393, Dec. 1963.

[94] L. B. White and B. Boashash, "Cross spectral analysis of nonstationary processes," IEEE Trans. Inf. Theory, vol. 36, no. 4, pp. 830-835, Jul. 1990.

[95] H. Cramér, "On some classes of nonstationary stochastic processes," in Proc. 4th Berkeley Symp. Mathematical Statistics and Probability. Berkeley, CA: Univ. Calif. Press, 1961, pp. 57-78.

[96] J. S. Bendat and A. G. Piersol, Measurement and Analysis of Random Data. New York: Wiley, 1966.

[97] J. K. Hammond and R. F. Harrison, "Wigner-Ville and evolutionary spectra for covariance equivalent nonstationary random processes," in Proc. IEEE Int. Conf. Acoustics, Speech and Signal Processing (ICASSP-85), Tampa, FL, Apr. 1985, pp. 1025-1028.

[98] P. Flandrin, "On the positivity of the Wigner-Ville spectrum," Signal Process., vol. 11, no. 2, pp. 187-189, 1986.

[99] L. L. Scharf, P. J. Schreier, and A. Hanssen, "The Hilbert space geometry of the Rihaczek distribution for stochastic analytic signals," IEEE Signal Process. Lett., vol. 12, no. 4, pp. 297-300, Apr. 2005.

[100] M. B. Priestley, Spectral Analysis and Time Series-Part II. London, U.K.: Academic Press, 1981

[101] W. A. Gardner, Statistical Spectral Analysis: A Nonprobabilistic Theory. Englewood Cliffs, NJ: Prentice-Hall, 1988.

[102] F. Hlawatsch and W. Kozek, "Time-frequency weighting and displacement effects in linear, time-varying systems," in Proc. IEEE Int. Conf. Acoustics, Speech and Signal Processing (ISCAS-92), San Diego, CA, May 1992, pp. 1455-1458.

[103] F. Hlawatsch and G. Matz, "Quadratic time-frequency analysis of linear time-varying systems," in Wavelet Transforms and Time-Frequency Signal Analysis, L. Debnath, Ed. Boston, MA: Birkhäuser, 2001, ch. 9, pp. 235-287.
[104] F. Hlawatsch and W. Kozek, "Second-order time-frequency synthesis of nonstationary random processes," IEEE Trans. Inf. Theory, vol. 41, no. 1, pp. 255-267, Jan. 1995.

[105] W. Mecklenbräuker and F. Hlawatsch, Eds., The Wigner DistributionTheory and Applications in Signal Processing. Amsterdam, The Netherlands: Elsevier, 1997.

[106] E. P. Wigner, "Quantum-mechanical distribution functions revisited," in Perspectives in Quantum Theory, W. Yourgrau and A. van der Merwe, Eds. New York: Dover, 1971, vol. 40, ch. 4, pp. 25-36.

[107] M. Loève, Probability Theory, 3rd ed. Princeton, NJ: Van Nostrand, 1962.

[108] W. Kozek, "Matched generalized Gabor expansion of nonstationary processes," in Proc. 27th Asilomar Conf. Signals, Systems, Computers, Pacific Grove, CA, Nov. 1993, pp. 499-503.

[109] — - "Optimally Karhunen-Loève-like STFT expansion of nonstationary random processes," in Proc. IEEE Int. Conf. Acoustics, Speech and Signal Processing (ICASSP-93), Minneapolis, MN, Apr. 1993, pp. 428-431.

[110] S. G. Mallat, A Wavelet Tour of Signal Processing. San Diego, CA: Academic, 1998.

[111] G. W. Wornell, "A Karhunen-Loève-like expansion for $1 / f$ processes via wavelets," IEEE Trans. Inf. Theory, vol. 36, no. 4, pp. 1534-1543, Jul. 1990.

[112] M. Unser, "An extension of the Karhunen-Loève transform for wavelets and perfect reconstruction filter banks," in Proc. SPIE Mathematical Imaging: Wavelet Applications in Signal and Image Processing, vol. 2034, Jul. 1993, pp. 45-56.

[113] A. J. E. M. Janssen, "Duality and biorthogonality for Weyl-Heisenberg frames," J. Fourier Anal. Applic., vol. 1, no. 4, pp. 403-436, 1995.

[114] G. Matz and F. Hlawatsch, "Linear time-frequency filters: Online algorithms and applications," in Applications in Time-Frequency Signal Processing, A. Papandreou-Suppappola, Ed. Boca Raton, FL: CRC, 2002, pp. 205-271.

[115] F. Hlawatsch and G. Matz, "Linear time-frequency filters," in Time-Frequency Signal Analysis and Processing: A Comprehensive Reference, B. Boashash, Ed. Oxford, U.K.: Elsevier, 2003, ch. 11.1, pp. 466-475.

[116] M. B. Priestley, "Filtering non-stationary signals," J. Roy. Statist. Soc. Ser. B, vol. 31, pp. $150-159,1969$. 